\title{
A Survey of Selected Aspects of Stratified and Rotating Fluids
}

\author{
Ronald G. Rehm \\ Institute for Basic Standards, National Bureau of Standards, Washington, D.C. 20234
}

(May 18, 1976)

A survey is presented of phenomena in stratified and rotating fluids. Care is taken to define important quantities, to discuss basic concepts, to derive the fundamental equations and to present the basic nondimensional parameters associated with these flows. After a rather extensive section on waves, the analogy between stratified and rotating flows is discussed. Then nonlinear processes and transport and diffusion processes are reviewed. Although this report is rather brief in parts, it displays the rich variety of phenomena in stratified and rotating fluids. It also tabulates many of the important references.

This report also contains a discussion of some applications. Physical oceanography, physical limnology and meteorology are all areas of application in the earth sciences. The variety of application is stressed at the expense of depth and completeness. The more technological application of plumes in bodies of water or in the atmosphere is also discussed.

Finally, some important problem areas are briefly surveyed. These areas are turbulence, nonlinear processes, numerical computation of flows, and further applications.

Key words: Buoyant flows; geophysical fluid flows; inertial waves; internal waves; rotating fluids; stratified fluids; waves in fluids.

\section{Contents}

Page

1. Introduction 354

2. General considerations _._. 355

2.1 Definitions and basic considerations

a. Equilibrium _... 355

b. Nonequilibrium 356

2.2 Basic parameters _...

a. Parameters in a stratified or rotating fluid 356

b. Dimensionless numbers

2.3 Typical magnitudes of characteristic parameters

2.4 Fundamental equations

a. The equations of conservation of mass and momentum _... 360

b. Equilibrium 361

c. The energy equation and the thermodynamic relations _...... 362

3. Small-Amplitude Waves _._.

3.1 General considerations 364

a. Hyperbolic waves _.

b. Dispersive waves

3.2 Methodology for Analysis of waves in dispersive, anisotropic media _... $\quad 370$

a. Kinematics of waves

b. Fourier analysis and asymptotics. 372

3.3 Waves in stratified fluids _

a. Derivation of the linearized equation for the vertical displacement

b. A survey of results on internal waves in a quiescent stratified fluid . $\quad 375$

3.4 Waves in rotating fluids

a. Derivation of equations _... 378

b. A survey of results on inertial waves in unconfined uniformly rotating fluids

c. Rossby waves

4. The analogy between stratified and rotating fluids

4.1 The stratification-dominated system, $S_{\Omega} \ldots 384$

4.2 The rotation-dominated system, $\Omega_{S}, \quad 385$

5. Nonlinear considerations _... 386

5.1 The equation of Long for stratified flow over an obstacle $\ldots \ldots$

5.2 The equation of Yih for stratified flow over an obstacle

5.3 Other nonlinear problems 
6. Transport and diffusion processes

6.1 Contained rotating fluids $\ldots \ldots \ldots$

6.2 Convection in stratified fluids

a. Buoyant thermals and plumes..... 394

b. Convection from heated surfaces

c. Double-diffusive convection

6.3 Turbulence $\ldots \ldots$

7. Some applications _._. 396

7.1 Physical oceanography _.

7.2 Physical limnology

7.3 Meteorology

7.4 Thermal discharges _.

8. Some important problem areas

9. Summary _.

10. References _.

\section{Introduction}

An increased interest in geophysical phenomena and greater public concern about the quality of the environment have led to an increase in activity in geophysical fluid dynamics. Although fluid-dynamical phenomena are only one aspect of such broader geophysical and environmental considerations, they constitute a very fundamental aspect. The atmosphere, after all, is the layer of fluid surrounding the earth in which we live and in which the flow and transport influence every aspect of our lives [1]. ${ }^{1}$ Also, the oceans and lakes are fluids covering: the major portion of the surface of the earth, and these also influence our living [2]. Even the dynamics of the solid surface of the earth is now thought to be dominated by convective motions within the earth, and these motions are being described by the plate-tectonic model of the earth [3].

Fluid phenomena within the atmosphere, oceans and lakes are often profoundly affected by the variations in density within the fluid $[4,5,6]$. Such fluids, where density varies as a function of depth, are called stratified fluids. As a result of the increased interest in geophysical flows, the study of stratified fluids has been re-awakened in recent years $[7,8]$. In a stratified fluid, buoyancy plays an important role, the stable configuration for the fluid being one in which the lighter, less-dense fluid overlays the heavier fluid. The ocean-atmosphere interface is an example of a stratified flow; here the very large density difference between the air and the underlying water is such that the inertial effects of the air are usually neglected in comparison with the inertia of the water. However, very generally, temperature variations and chemicalcomposition and solid-particle variations provide density stratifications which lead to subtle and often profound differences in the flow and transport phenomena.

A second effect within geophysical flows which can profoundly influence the flow and which has also undergone a resurgance of interest is the effect of rotation of the earth $[9,10]$. The influence of rotation generally applies to larger scale phenomena than those associated with stratification, but often flows are affected by both rotational and stratification effects simultaneously $[6,11,12,13]$. The effects of stratification and rotation are not only important in geophysical applications, but also in astrophysical applications [14, 15].

There is an analogy between the effects of rotation and those due to stratification [16]. Rotation of a homogeneous fluid about the vertical axis inhibits the flow in horizontal planes. Similarly, in a stable, density-stratified fluid, motion of the fluid in the vertical direction (parallel to the direction of gravity) is inhibited because of the buoyancy variation of the fluid. Under certain conditions the analogy reduces mathematically to the same problem. For wave phenomena the analogy has been known for some time, and a mathematical formalism has been developed to analyze these waves (as well as those arising in other flows such as magnetohydrodynamic flows [17]).

In this paper an exposition is given of stratified and rotating phenomena. There are several excellent texts which have come out recently (refs. [4, 5, 6, 7] and [9]) which examine stratified or rotating fluids, and this paper will rely heavily on these texts. However, additional features will also be considered.

In the second section general considerations, such as definitions of terms, basic concepts, basic parameters and the fundamental equations, in the study of stratified and rotating fluids

\footnotetext{
${ }^{1}$ Figures in brackets indicate the literature references at the end of this paper.
} 
will be presented. In the third section an examination of small disturbances (waves) to equilibrium configurations will be discussed. Because of the interest of the author in this area, this section will be rather extensive. In section four the analogy between stratified and rotating fluids is discussed in some detail. Nonlinear features of stratified or rotating flows are presented in section five, and transport and diffusion processes in these flows are surveyed in section six. Section seven deals with applications of stratified and rotating flows to oceanography, limnology, meteorology, and thermal discharges. Because the fields of application are extremely broad, this section will not be very detailed. Finally, section eight will present some important problems in the study of phenomena in stratified and rotating flows, and section nine will give a summary and conclusions.

\section{General Considerations}

In this section a discussion is presented of the basic fluid-dynamical considerations for stratified and/or rotating fluids. This discussion will attempt to be simple and clear and yet to introduce all of the important aspects of these systems. Reference to the several excellent texts on these subjects will be made frequently $[4-7,9]$.

\subsection{Definitions and Basic Considerations}

For all systems of fluids the basic relations which determine the dynamics are the conservation of mass, momentum and energy. These relations are then supplemented by others which relate various fluid properties such as the equation of state, a relation between stress and strain rate, etc. The equations which express these relations will be presented in another subsection. The purpose of this subsection will be to define fundamental quantities and to introduce basic concepts related to stratified and rotating fluid systems.

\section{a. Equilibrium}

A stratified fluid is one in which the fluid is not homogeneous throughout, but in equilibrium resides in layers or strata, each of which has different physical properties. The physical property which most affects the dynamical behavior of the fluid is the density. Yih [7] points out that the density stratification between layers has two important effects, the inertia effect and the gravity effect. Since the inertia of a fluid element is proportional to its density, a density variation produces an inertia variation.

When a body force (gravity) is present within the fluid, then the variation of density also affects the body force on different fluid elements. In equilibrium because of the downward gravitational body force, a fluid will establish itself in a stable configuration so that the fluid of greatest density is at bottom while the least dense fluid will be at the top. The gravity effect of the stratification will be the feature of stratified fluids which is emphasized in this paper, but inertia effects will also be discussed.

In a stably stratified fluid, displacement upward of a small fluid element from its equilibrium position leads to a downward gravitational restoring force because the density of the parcel is greater than that of the fluid at its displaced height. Therefore, the fluid parcel will be heavier than its surroundings and will tend to sink back toward its equilibrium height. Similarly, displacement downward of the parcel leads to it being less dense than its surrounding: fluid, and an upward buoyancy force tends to restore it. Because of this restoring bouyancy force, the fluid is stable.

The argument given above applies to a fluid in which the density does not change when displaced, an incompressible fluid. An essential concept when considering a compressible fluid is that of potential density (or potential temperature). When a fluid element is displaced, it experiences a change in pressure, and, corresponding to this change in pressure, a change in density (or temperature). If no dissipative mechanisms act, the change in pressure results in an adiabatic change in density (temperature). The potential density (temperature) is defined as the density (temperature) resulting when the fluid is compressed adiabatically to a standard pressure. For the atmosphere, a perfect gas is a good approximation to the equation of state and the potential density (and temperature) is important because of the compressibility of the gas. (See reference [4] for a more detailed discussion of potential density and temperature.)

In a rotating fluid the equilibrium configuration is likewise simple, a state of rigid-body rotation. In this case the fluid may be described in a reference coordinate system which rotates with the fluid, and in this reference frame the fluid is at rest. The fluid in rotation is also stable; here the angular momentum of the fluid provides a mechanism for stabilization. When a fluid element is displaced outward from its equilibrium radius, its angular momentum will remain constant. Since it will have a smaller angular momentum than the surrounding fluid at its displaced radius, the Coriolis (or centrifugal )force will tend to restore it to its equilibrium radius. 
Similarly, if the fluid element is displaced inward, its excess angular momentum will again tend to restore it to its equilibrium radius.

\section{b. Nonequilibrium}

When a stratified or rotating fluid is altered from its equilibrium state, a variety of nonequilibrium processes can occur. Although these processes can perhaps be classified in other ways, in this survey they will be classified as nondissipative and dissipative. Nondissipative flows are ones in which the effects of viscosity and thermal conductivity, for example, can be neglected. Turbulent mixing, which behaves as a diffusion process, will also be excluded in consideration of such flows. Dissipative flows, therefore, are ones where some dissipative mechanism determines the structure of the flow. Boundary layers are a prime example of such flows.

Nondissipative flows will further be broken down into linear flows (or waves) and nonlinear flows. In a very interesting and penetrating review article [18], Lighthill has surveyed the different types and the nature of waves in fluids. The study of waves is very important for two reasons. First the study of waves in an otherwise steady state is a study of linear phenomena and linear analysis is highly developed compared with nonlinear analysis. Therefore, most of the information we acquire mathematically about a system is from the analysis of small disturbances. Second, and not entirely unrelated to the first, is that essential physical mechanisms of a system come to light in the study of waves. Some nonlinear phenomena will also be considered in this survey.

In an inviscid, incompressible fluid with no body forces, waves can be sustained only in the presence of a preexisting flow or in a two-component system [18]. When a gravitational body force is present in a quiescent homogeneous fluid, waves can exist at a surface only; classical gravitational surface waves are the examples of these waves. In a stratified fluid, however, waves can be sustained for which the amplitude is largest within the fluid and not at its surface. Such waves are called internal waves. Likewise, because of the effective body force (Coriolis force) in a rotating fluid, waves can be sustained internally to the fluid. Such waves are known as inertial waves. In this survey internal and inertial waves will be discussed in some detail

\subsection{Basic Parameters}

\section{a. Parameters in a Stratified or Rotating Fluid}

In a stratified fluid the basic parameter which describes the stability of the fluid is the Brunt-Väisälä frequency. As discussed in the previous subsection, a fluid element displaced either upward or downward from equilibrium experiences a restoring force. This force is directly proportional to the acceleration of gravity $g$ and to the equilibrium density gradient

$$
\frac{d \rho_{0}}{d z}, \text { where } \rho_{0}
$$

is the equilibrium density distribution (only a function of $z$ ), $z$ is measured positive upward and gravity acts downward. The Brunt-Väisälä frequency $N$ is proportional to the square root of this restoring force and is the frequency with which the fluid parcel would oscillate about its equilibrium position if initially displaced. The expression for $N$ is

$$
N=\left(-\frac{\frac{d \rho_{0}}{d z}}{\rho_{0}} g\right)^{1 / 2}
$$

When there is no density variation with depth (a homogeneous fluid), the Brunt-Väisälä frequency vanishes and the fluid is neutrally stable.

In general the Brunt-Väisälä (B-V) frequency is a function of depth within the fluid. In the ocean, for example, the $\mathrm{B}-\mathrm{V}$ frequency has a very characteristic behavior as a function of depth, being rather small in a well mixed upper layer and at great depths, but rising to a sharp peak generally at a depth of some hundreds of meters $[5,6]$. The depth at which the B-V frequency is maximum is called the thermocline and basically divides two layers of fluid in each of which the fluid is nearly homogeneous. In large lakes the B-V frequency is usually similar in form to that in the ocean, with the thermocline occurring at much smaller depths $[6,19]$. 
The basic stability considerations are changed somewhat when the fluid is compressible, as discussed in the preceding subsection. In a compressible fluid, acoustic as well as internal waves can be sustained within the fluid, each type of wave depending upon different mechanisms. A basic parameter which arises in the dynamics of a compressible fluid is the speed of sound $c$, the speed with which an acoustic signal propagates [20]. When the compressibility of the fluid is also taken into account in a stratified fluid, the Brunt-Väisälä frequency must be modified to be

$$
N=\left[-g\left(\frac{\frac{d \rho_{0}}{d z}}{\rho_{0}}+\frac{g}{c^{2}}\right)\right]^{1 / 2}
$$

In the atmosphere, where the compressibility must generally be accounted for, the B-V frequency generally passes through two minima and two maxima with increasing altitude [6].

In a rotating fluid the corresponding parameter is the angular velocity $\Omega$ of rotation of the

fluid. In this case a fluid parcel displaced from its equilibrium radius will oscillate at twice the angular velocity about its equilibrium position. Therefore, the parameter $2 \Omega$ serves the same purpose within a rotating fluid as the stability parameter $N$ does in a stratified fluid.

\section{b. Dimensionless Numbers}

Several nondimensional parameters arise in the study of stratified and rotating flows. These parameters can be divided into those which describe local properties within the flow (and therefore vary from point to point within the fluid) and overall dimensionless numbers which characterize the whole flow. (Batchelor [21] discusses this difference in some detail.) In this paper mainly the latter type of dimensionless parameter will be discussed. (See references [4] and [9] for more complete discussions of dimensionless numbers in stratified and rotating fluids.)

To define overall dimensionless numbers it is necessary to select characteristic scales of velocity $U$, length $L$, density $\rho_{0}$ and density variation $\Delta \rho_{0}$ for the flow. (With this notation an overall Brunt-Väisälä frequency can be defined as

$$
N_{0}^{2}=\frac{g \Delta \rho_{0}}{\rho_{0} L}
$$

An obvious dimensionless number characterizing the stratification of the fluid is the density ratio $n=\Delta \rho_{0} / \rho_{0}$.

A second dimensionless number, which will be used in this paper is the Richardson number, defined as

$$
R i=\frac{g \Delta \rho_{0} L}{\rho_{0} U^{2}} .
$$

The Richardson number compares the buoyancy to the inertia terms in the momentum conservation relations. When the motions are steady, the effect of variations in density in the inertia terms can be ignored (the Boussinesq approximation) and the flow is nondissipative, then the Richardson number is the only dimensionless number characterizing the flow [4].

Often the Froude number is used to characterize surface flows; the Froude number is defined as

$$
\operatorname{Fr}=\frac{U}{(g L)^{1 / 2}} .
$$

It compares a characteristic flow velocity to the velocity of long waves on a free surface [4]. For stratified flows the internal, or densimetric Froude number, defined as

$$
F=\frac{U}{\left(\frac{\Delta \rho_{0}}{\rho_{0}} g L\right)^{1 / 2}}
$$

is often used instead of the Richardson number. The densimetric Froude number is the inverse square root of the Richardson number. In this paper the Richardson number will be used consistently. 
When the flow is dissipative, additional dimensionless parameters can be defined because additional physical effects also become important. In a flow where viscosity is important, the Reynolds number is also a relevant parameter.

$$
R e=\frac{U L}{\nu}
$$

where $\nu$ is the kinematic viscosity. As is well-known, the Reynolds number compares the inertia to viscous effects. In geophysical flows, it is generally very large, indicating that boundary layers may be important and that turbulence is probably a dominant feature in these flows [5].

Another fundamental number is the Prandtl number, defined as

$$
\operatorname{Pr}=\nu / \kappa
$$

where $\kappa$ is the thermometric diffusivity. The Prandtl number compares the viscous diffusion of momentum to the diffusion of heat.

In a rotating fluid the key parameter in the absence of dissipative effects is the Rossby number, defined by

$$
\epsilon=\frac{U}{\Omega L} \text {. }
$$

It is the ratio of the convective acceleration to the Coriolis acceleration and also provides an estimate of the importance of nonlinear effects [9]. When $\epsilon<<1$, linear theories are appropriate, whereas nonlinear analysis must be used when $\epsilon$ approaches unit magnitude.

Dissipative effects of viscosity are measured by means of the Ekman number, defined as

$$
E=\frac{\nu}{\Omega L^{2}}
$$

which compares the viscous force to the Coriolis force. It is generally quite small, indicating: the potential importance in rotating fluids of boundary layers.

When both rotation and stratification are important, both sets of dimensionless parameters must be considered. Other nondimensional numbers can be formed from these basic ones and will be introduced as needed.

When time-dependent phenomena, are considered, a frequency of oscillation $\omega$ is also a relevant parameter. Then two additional nondimensional numbers $\omega / N_{0}$ and $\omega / 2 \Omega$ will also be important. The first is the ratio of the frequency of oscillation within the flow to the characteristic B-V frequency while the second is the ratio of the frequency of oscillation to twice the rotational frequency of the fluid. These ratios will be important when wave propagation is considered in section three.

When the compressibility of the fluid is important, the velocity of sound $c$ also becomes a relevant parameter. Generally, the sound speed varies from point to point within the fluid. A nondimensional number describing the flow with compressibility effects is the Mach number

$$
M=U / c_{0}
$$

where $c_{0}$ is a characteristic sound speed (say the maximum value). Generally, in stratified and rotating fluids the compressibility is neglected.

\subsection{Typical Magnitudes of Characteristic Parameters}

In this subsection a tabulation is presented of the magnitudes of the parameters discussed in the preceding subsection. These estimates provide a guide to analyses of stratified or rotating flows since they indicate which physical effects can be expected to be important in various flows.

Table 1 presents for reference the values of the acceleration of gravity and the angular velocity of the earth. Then a list of representative values of the basic physical properties for air and water are tabulated. Since these properties are functions of temperature and pressure, in general, it is necessary to determine the temperature range and pressure range of interest before appropriate values can be determined. These values have simply been listed for reference.

Also listed in table 1 is a range of values of the Brunt-Väisälä frequency and period for the ocean, for lakes and for the atmosphere. From this tabulation it is apparent that there is a wide variation in the values of this parameter and, consequently, a wide variation in the 
effect of stratification. Finally, the magnitude of the density variation across the thermocline in the ocean or in lakes is given in this table.

Using values in this table and values of appropriate length and velocity scales, estimates of the magnitudes of the nondimensional numbers can be made. First, the ratio of density variation across the thermocline to absolute density is approximately $n \cong 10^{-3}$.

TABLE 1.-Representative values of parameters

\begin{tabular}{c|c}
\hline \hline Acceleration of Gravity and Angular Velocity of the Earth \\
\hline Parameter & \multicolumn{1}{|c}{ Value } \\
\hline$g$ & $981 \mathrm{~cm} / \mathrm{s}$ \\
$2 \Omega$ & $1.45 \times 10^{-4} \mathrm{rad} / \mathrm{s}$ \\
$T_{\Omega}=2 \pi / \Omega$ & $24 \mathrm{~h}$ \\
\hline
\end{tabular}

Basic Physical Properties of the Fluid

\begin{tabular}{|c|c|c|c|}
\hline \multicolumn{2}{|c|}{ Water } & \multicolumn{2}{|c|}{ Air } \\
\hline Parameter & Value & Parameter & Value \\
\hline $\begin{array}{l}\rho_{0}\left(4^{\circ} \mathrm{C}\right) \\
\mu\left(20^{\circ} \mathrm{C}\right) \\
\nu=\mu / \rho_{0} \\
c_{0} \\
k=\text { thermal conductivity } \\
C_{\mathrm{p}} \\
\kappa=k / \rho C_{\mathrm{p}} \\
\operatorname{Pr}=\nu / \kappa\end{array}$ & $\begin{array}{l}\text { 1. } 0 \mathrm{~g} / \mathrm{cm}^{3} \\
1.0 \times 10^{-2} \mathrm{~g} / \mathrm{cm}-\mathrm{s} \\
1.0 \times 10^{-2} \mathrm{~cm}^{2} / \mathrm{s} \\
\text { 1. } 5 \times 10^{5} \mathrm{~cm} / \mathrm{s} \\
5.8 \times 10^{-3} \mathrm{~W} / \mathrm{cm}-\mathrm{K} \\
\text { 4. } 18 \mathrm{~J} / \mathrm{g}-\mathrm{K} \\
\text { 1. } 4 \times 10^{-3} \mathrm{~cm}^{2} / \mathrm{s} \\
7.2\end{array}$ & $\begin{array}{l}\rho_{0}\left(0^{\circ} \mathrm{C}, 1 \mathrm{~atm}\right) \\
\mu\left(20^{\circ} \mathrm{C}\right) \\
\nu=\mu / \rho_{0} \\
c_{0} \\
k \\
C_{p} \\
\kappa \\
\operatorname{Pr}\end{array}$ & $\begin{array}{l}\text { 1. } 29 \times 10^{-3} \mathrm{~g} / \mathrm{cm}^{3} \\
1.81 \times 10^{-4} \mathrm{~g} / \mathrm{cm}^{-\mathrm{s}} \\
1.36 \times 10^{-1} \mathrm{~cm}^{2} / \mathrm{s} \\
\text { 3. } 5 \times 10^{4} \mathrm{~cm} / \mathrm{s} \\
\text { 2. } 4 \times 10^{-4} \mathrm{~W} / \mathrm{cm}^{-\mathrm{K}} \\
\text { 1. } 0 \mathrm{~J} / \mathrm{g}-\mathrm{K} \\
1.80 \times 10^{-1} \mathrm{~cm}^{2} / \mathrm{s} \\
\text { 7. } 6\end{array}$ \\
\hline
\end{tabular}

Range of Values of the Brunt-Vaisala Frequency and Period

\begin{tabular}{|c|c|c|c|}
\hline & Ocean & Lake & Atmosphere \\
\hline $\begin{array}{l}N \\
T_{N}=2 \pi / N \\
\Delta \rho_{0} \text { (across thermocline) }\end{array}$ & $\begin{array}{l}10^{-2}-10^{-4} \mathrm{rad} / \mathrm{s} \\
10 \mathrm{~min}-2 \mathrm{~h} \\
10^{-3} \mathrm{~g} / \mathrm{cm}^{3}\end{array}$ & $\begin{array}{l}5 \times 10^{-2}-10^{-4} \mathrm{rad} / \mathrm{s} \\
2 \mathrm{~min}-2 \mathrm{~h} \\
10^{-3} \mathrm{~g} / \mathrm{cm}^{3}\end{array}$ & $\begin{array}{l}3 \times 10^{-2}-10^{-2} \mathrm{rad} / \mathrm{s} \\
3 \mathrm{~min}-10 \mathrm{~min}\end{array}$ \\
\hline
\end{tabular}

Values of the Richardson number (or the internal Froude number) depend upon the values of the velocity scale $U$ and the length scale $L$. In meteorological applications, velocities are typically measured in meters per second or greater and lengths are measured in meters or greater (kilometers to thousands of kilometers). In oceanographic applications velocities are typically much smaller, being measured in centimeters per second with lengths comparable to those in atmospheric considerations. Therefore, the Richardson number is typically rather small, ranging from values at most of order unity down to values of order $10^{-4}$ or smaller.

Here, it should be noted that the vertical length and velocity scales are characteristically smaller than the corresponding horizontal scales. Because the oceans, lakes and the atmosphere are relatively shallow, but laterally extensive bodies of fluid and because the stratification inhibits only the vertical motion, the characteristic vertical length and velocity are often much smaller than the corresponding horizontal quantities. In the Richardson number, many times the relevant length scale is the vertical one while the velocity scale is the horizontal one.

In the laboratory, typical length and velocity scales are much smaller while the density variation is generally comparable to that in geophysical fluids $[22,23]$. Because the velocity scale appears quadratically in the denominator while the length appears only linearly in the numerator, the Richardson number is characteristically much larger. If the velocity is measured in centimeters/second and the length in centimeters, then the Richardson number is of order unity.

The Reynolds number varies even more dramatically between geophysical and laboratory flows. Since the kinematic viscosity varies by over an order of magnitude between water and air, 
characteristic Reynolds numbers will also vary between oceans and lakes on the one hand and the atmosphere on the other. In oceans or lakes, taking a length scale of one meter and a velocity of one meter per second, the Reynolds number is of order $10^{6}$. In the atmosphere, with the same length and velocity scale, $\cong 10^{5} \mathrm{Re}$. In the laboratory, taking a length of one centimeter and a velocity of one centimeter per second, in water, $\mathrm{Re} \cong 10^{2}$ whereas in air $\mathrm{Re} \cong 10$.

The Prandtl number is a characteristic of the fluid and therefore has been listed in table 1. In a rotating fluid the Rossby number can be calculated for geophysical fluids and laboratory fluids. In geophysical applications, a velocity of a meter per second and a length of a meter yield a Rossby number, which is very large; hence these scales are not consistent since the Rossby number must be of order unity or smaller [9]. The velocity must be smaller or the length longer to make the Rossby number of order unity; values of $U=10 \mathrm{~cm} / \mathrm{s}$ and $L=10^{5} \mathrm{~cm}$ would be much more appropriate. In the laboratory representative values given by Greenspan [9], are $\Omega=1 \mathrm{rad} / \mathrm{s}, L=20 \mathrm{~cm}$ and $\epsilon=0.01($ or $U=0.2 \mathrm{~cm} / \mathrm{s})$.

The Ekman number is of order $10^{-8}$ for oceans or lakes when a length of $10^{5} \mathrm{~cm}$ and the viscosity of water is used. Using the same length scale and the viscosity of air, in the atmosphere the Ekman number is of order $10^{-7}$. (It should be noted that many analyses are conducted using a much larger kinematic viscosity to represent the fact that the motion is turbulent. This would change the value of the Ekman number considerably, although it would probably still remain small.) For the laboratory, using $\Omega=1 \mathrm{rad} / \mathrm{s}$ and $L=20 \mathrm{~cm}$ and water, the Ekman number is of order $2 \times 10^{-5}$. Therefore, in all cases the Ekman number is very small, implying: that viscous effects in the main body of the flow are small and that boundary layers will arise.

Table 2 presents a list of nondimensional numbers calculated in this subsection. The Mach number, based upon a velocity of one meter per second and the sound speeds given in table 1, is seen to be very small.

TABLE 2.-Representative values of dimensionless numbers

\begin{tabular}{l|c|c|c}
\hline \hline \multicolumn{2}{c|}{ Dimensionless Number } & \multicolumn{2}{|c}{ Value } \\
\hline & Lakes \& Oceans & Atmosphere & Laboratory \\
\cline { 2 - 4 } & $10^{-3}$ & & $10^{-3}$ \\
$n$ & $1-10^{-4}$ & & $10-10^{-1}$ \\
$R i$ & $10^{6}$ & $10^{5}$ & $10^{2}$ \\
$P r$ & 1.2 & 7.6 & 7.2 \\
$\epsilon$ & 1 & 1 & 0.01 \\
$E$ & $10^{-8}$ & $10^{-7}$ & $2 \times 10^{-5}$ \\
$M$ & $<10^{-2}$ & $<10^{-2}$ & $<10^{-2}$ \\
\hline
\end{tabular}

\subsection{Fundamental Equations}

The fundamental equations are expressions of conservation laws for mass, momentum and energy. These equations are derived in various forms in each of the primary references $[4,5$, $6,7]$ and [9]. Therefore, in this subsection, the equations will simply be stated in suitable form and some observations will be made concerning them.

\section{a. The Equations of Conservation of Mass and Momenfum}

The conservation of mass for a compressible fluid is written in standard form as

$$
\frac{\partial \rho}{\partial t}+\nabla \cdot(\rho \mathbf{u})=0
$$

where $\rho$ is the density of the fluid, $\mathbf{u}$ the velocity, $t$ time and the coordinates are $x_{1}, x_{2}$, and $x_{3}$ (or sometimes $x, y$, and $z$ will be used). This equation can be written in several alternate forms, and these forms will be used as needed.

The equation for the conservation of momentum is somewhat different than the standard form written in most introductory texts on fluid mechanics. Because the fluid is stratified and gravity $g$ acts as a body force per unit mass, the equations must include an expression for this 
body force. In addition, when a rotating fluid is considered, this equation is written with respect to a reference frame that rotates with the fluid at a constant angular velocity $\Omega$. This equation is

$$
\rho \frac{D \mathbf{u}}{D t}+2 \rho \boldsymbol{\Omega} \times \mathbf{u}+\rho \boldsymbol{\Omega} \times(\boldsymbol{\Omega} \times \mathbf{r})+\nabla p-\rho \mathbf{g}=\mathbf{f} .
$$

Here, $p$ is pressure, $\mathbf{r}=\left(x_{1}, x_{2}, x_{3}\right)$ is the radius vector to a fluid element and $\mathbf{f}$ is the resultant of all other forces acting on a unit volume of fluid. Both the angular velocity $\boldsymbol{\Omega}$ and the gravitational body force $\mathrm{g}$ have been made into vector quantities since each has a direction. The time derivative is the substantial derivative

$$
\frac{D}{D t}=\frac{\partial}{\partial t}+\mathbf{u} \cdot \nabla
$$

The two terms involving $\Omega$ in the equation represent the effective body force per unit volume due to the rotation. The first term is the Coriolis force while the second is usually called the centrifugal force. Often the centrifugal-force term is absorbed in the pressure term by defining a reduced pressure. (See ref. [9] for details.)

The terms on the right-hand side will generally be taken to be a result of viscous forces alone. When viscous stresses are considered, it will also be assumed that the fluid is incompressible and $\mathbf{f}=\rho_{0} \nu \nabla^{2} \mathbf{u}$ will be used. Otherwise it will be assumed that the right-hand size is zero.

\section{b. Equilibrium}

In equilibrium the simplest case to consider is when the fluid is in a steady state so that $\frac{\partial}{\partial t}=0$, and there is no velocity of the fluid in the rotating reference frame. Then

$$
\rho_{0} \boldsymbol{\Omega} \times(\boldsymbol{\Omega} \times \mathbf{r})+\nabla p_{0}-\rho_{0} \mathbf{g}=0 .
$$

Two basic equilibrium states will be considered, the state of no rotation in a stratified fluid and the state of no stratification in a rotating fluid.

When there is no rotation, the equilibrium is determined by this equation with the first term set to zero, the so-called hydrostatic equilibrium state:

$$
\nabla p_{0}=\mathbf{g} \rho_{0} .
$$

In this case the pressure gradient is parallel to the gravitational body force and then the density and pressure depend upon one coordinate parallel to $\mathbf{g}$.

The other basic equilibrium state is one in which the fluid is unstratified but rotating. In this state the last term in eq (13) is zero, and the pressure gradient is balanced by the centrifugal force. A great deal can be learned about the character of flows which deviate somewhat from this state of equilibrium by a fundamental theorem for rotating flows called the TaylorProudman Theorem [9, 72]. The Taylor-Proudman theorem states that when the flow in a rotating reference frame is steady, inviscid and slow (so that the Rossby number is small, $\epsilon<<1$ ), then the motion does not vary in the direction of the rotation vector. Howard [72] has given a very clear and concise discussion of this theorem together with a discussion of other important ideas such as Taylor columns, geostrophic flow and deviations from geostrophic flow. The following presentation follows his rather closely.

For an incompressible fluid, the equation of continuity (11) reduces to the statements that $\nabla \cdot \mathbf{u}=0$ and $\rho$ is constant following a fluid element. For slow, steady and inviscid flow, eq (12) reduces to the equation $[9,72]$

$$
2 \boldsymbol{\Omega} \times \mathbf{u}=-\nabla(p / \rho-1 / 2(\boldsymbol{\Omega} \times \mathbf{r}) \cdot(\boldsymbol{\Omega} \times \mathbf{r})+g z) .
$$

The Taylor-Proudman theorem follows directly by taking the curl of this relation and noting that $\boldsymbol{\Omega}$ is constant and $\nabla \cdot \mathbf{u}=0$ :

$$
\boldsymbol{\Omega} \cdot \nabla \mathbf{u}=0
$$

Therefore there is no variation in the velocity in the direction of the rotation. 
Equation (13), representing a balance between the (reduced) pressure force and the Coriolis force, is known as the "geostrophic approximation" [1, 6, 9, 72]. When the rotation and buoyancy force are taken as vertical, then the vertical component of eq (13) is simply the hydrostatic equilibrium equation, eq $(12 \mathrm{~b})$. The horizontal components of this equation then yield the balance between pressure and Coriolis forces.

$$
\begin{array}{r}
-2 \Omega \rho u=-\frac{\partial p}{\partial x} \\
2 \Omega \rho v=-\frac{\partial p}{\partial y}
\end{array}
$$

where $u$ and $v$ are the $x$ and $y$ or horizontal components of the velocity, and $p$ is the reduced pressure [9].

Equations (14) show that [72]

$$
v \frac{\partial p}{\partial x}+u \frac{\partial p}{\partial y}=0
$$

Hence the velocity vector is perpendicular to the pressure gradient in the horizontal plane, or the stream lines coincide with constant-pressure contours (isobars). Many large-scale geophysical flows are approximately geostrophic [6].

If the fluid is bounded by a horizontal surface (perpendicular to the axis of rotation) as would be the case in the laboratory for example, then the vertical velocity must be zero at this surface. By the Taylor-Proudman theorem, then, the vertical velocity is zero throughout so that the flow is purely two-dimensional and horizontal.

Taylor [73] demonstrated the validity of these considerations with an experiment which is well known now. In this experiment he slowly dragged across the bottom of a rotating tank a small circular bump. In the absence of motion of the bump, the fluid in the tank rotates as a solid body. But when the bump is dragged slowly across the bottom, all motions relative to the tank are two dimensional so that the column of fluid directly above the bump is dragged along above the bump. This column (and similar columnar flow) has become known subsequently as a Taylor column, and a great deal of effort has been expended to understand how such columns form and their structure [9].

\section{c. The Energy Equation and the Thermodynamic Relations}

The energy conservation equation can be written in a variety of ways, and is usually written in a form suitable for the particular application. A general and convenient form is

$$
\frac{D s}{D t}=q / T
$$

where $s$ is the entropy per unit mass, $q$ is the energy added per unit mass per unit time, $T$ is the temperature and $D / D t$ is the substantial derivative.

The dominant form of energy addition to a fluid element will be taken to be heat conduction. Viscous degradiation of mechanical energy also adds heat to an element, but this energy addition is small $[6,9]$ and will not be considered. Radiative energy addition in the atmosphere and at the surface of water bodies is important, but will not be examined here. When heat conduction is important, the energy addition term is

Otherwise $q$ will be taken to be zero.

$$
q=\frac{1}{\rho} \nabla \cdot(k \nabla T) .
$$

From thermodynamics relations eq (16) can be written in other forms; one particular form which will be useful later is

$$
\rho C_{p} \frac{D T}{D t}-\alpha T \frac{D p}{D t}=\rho q
$$


where $C_{p}$ is the constant-pressure specific heat and

$$
\alpha=-\frac{1}{\rho}\left(\frac{\partial \rho}{\partial T}\right)_{p=\text { constant }}
$$

is the coefficient of thermal expansion. As noted by Greenspan [9], the second term on the left is generally small in liquids. If this term is neglected and $k$ is assumed constant, then eq (18) reduces to

where

$$
\frac{D T}{D t}=\kappa \nabla^{2} T
$$

$$
\kappa=k / \rho C_{p} .
$$

In this equation convection and thermal diffusion are important for determination of the temperature distribution.

Different equations of state and thermodynamic relations will be considered depending upon the fluid being considered. The atmosphere is a mixture of gases, predominantly nitrogen and oxygen; but in the atmosphere an equation of state appropriate for a single perfect gas of average molecular weight 29 can be used. Hence

where

$$
p=\rho R T
$$

$$
R=2.87 \times 10^{2} \mathrm{~J} / \mathrm{kg}-K
$$

can be used with the ratio of specific heats $\gamma=1.4$ (ref. [6]). The other thermodynamic relations are also those appropriate to a perfect gas.

Some limitations to the use of these equations should be noted. First, the atmosphere contains water vapor, and this water vapor, through the mechanisms of evaporation and condensation, has major effects upon the dynamical processes. Water vapor and other constituents of the atmosphere, most notably carbon dioxide, also play a fundamental role in the thermal balance of the atmosphere through absorption and re-radiation. (The temperature variation in the atmosphere is determined in large part by the incident solar radiation and by the low-temperature radiation from the surface of the earth.) The atmosphere contains particulate matter, and this material is also important. Finally, at higher altitudes the assumptions of a diatomic, neutral gas may break down since the gas dissociates or ionizes. (At even higher altitudes the assumptions that the gas is a continuum breaks down.) However, none of these considerations will be taken into account in this report.

In lakes the equation of state and thermodynamic relations appropriate to fresh water can be used [6]. The only point to be noted with respect to these relations is that the basic coefficients in various relations are not constant. Rather, water at atmospheric pressure has its maximum density at about $4^{\circ} \mathrm{C}$, and the temperature at which the maximum density occurs is a function of pressure Therefore, the coefficient of thermal expansion for water vanishes at a certain temperature, which itself is a function of pressure. This variation has important implications for the stratification cycle of lakes $[6,22]$. The equation for the variation in the coefficient of thermal expansion in pure water can be taken as [6]

$$
\alpha \equiv \frac{-1}{\rho}\left(\frac{\partial \rho}{\partial T}\right)_{p}=15 \times 10^{-6} T_{M}
$$

where

$$
T_{M}=T-4.0+2 \times 10^{-8} p
$$

with $p$ given in atmospheres and $T$ in degrees Celsius. The density variation from a reference density is then taken to be proportional to the variation in temperature from the reference temperature with the coefficient for thermal expansion being the proportionality factor.

In the ocean, salinity plays a very important role in the thermodynamic relations. The salinity is the mass of dissolved solids per unit mass of sea water. Once again, the coefficients in these relations cannot be taken as constants: for example, the coefficient of thermal expansion can be expressed as

$$
\alpha=9.5 \times 10^{-6} T^{\prime}{ }_{M}
$$


where

$$
T^{\prime}{ }_{M}=T+7.0+2 \times 10^{-9} p
$$

with $p$ given in atmospheres and $T$ in degrees Celsius.

In general, the salinity of the water is not constant from position to position, and an equation of state for sea water should consider salinity, as well as two other basic thermodynamic variables (such as pressure and temperature), to be a basic thermodynamic variable. If this procedure is followed, an equation of continuity for the salinity and other equations relating the flux density of salt to the temperature and salinity gradients must be added to the basic equations.

When salinity gradients are considered, it can often be assumed that the density can be expressed as a linear function of the variation in salinity from some reference value. Therefore, with $\rho_{\infty}$ the reference value of density and $T_{\infty}$ and $S_{\infty}$ the reference values of temperature and salinity, the complete variation of density can be expressed as

$$
\rho=\rho_{\infty}\left[1-\alpha\left(T-T_{\infty}\right)+\beta\left(S-S_{\infty}\right)\right]
$$

where

$$
\alpha \text { and } \beta \equiv \frac{1}{\rho}\left(\frac{\partial \rho}{\partial S}\right)_{T, p}
$$

are "the coefficients of expansion." The energy equation will be taken to be eq. (19), and the equation for salinity conservation will likewise have this form

$$
\rho \frac{D S}{D t}-\nabla \cdot(\rho D \nabla S)=0
$$

where $D$ is the diffusion coefficient for salinity $[4,5]$.

A brief observation should be made regarding the Boussinesq approximation. If the compressibility of the fluid can be neglected (as is generally the case in water and often the case in the atmosphere), the Boussinesq approximation can be made [5]. In this approximation, variations in density from a constant reference density are considered to be significant only in buoyancy terms (that is, terms multiplied by the acceleration of gravity). In inertia terms the actual density is replaced by the reference value. This approximation is generally excellent and will be used except in a few parts of this report.

\section{Small-Amplitude Waves}

Lighthill [18] has presented a survey of the different types and the nature of waves in fluids. In that survey he discussed the physical mechanisms for wave phenomena and some mathematical aspects of the formulation and solution for such waves. Furthermore, in a series of papers $[17,18,24,25]$ he has presented a mathematical formalism for the solution of the equations of a general class of these wave motions. A recent book by Whitham [26] presents a detailed and rather complete discussion of waves, the formulation of equations describing these waves and methodology for solution of the equations.

In this section a discussion of waves will be presented which relies heavily on the papers and monograph referenced above. The emphasis of this discussion will be on waves in stratified and rotating fluids. In the first subsection general considerations of waves will be presented including the classification of these waves into hyperbolic and dispersive classes. In the second subsection the general methods will be discussed for analysis of equations describing smallamplitude waves. In the final two subsections waves in stratified and rotating fluids respectively will be surveyed.

\subsection{General Considerations}

Whitham [26] defines a wave as "any recognizable signal that is transferred from one part of the medium to another with a recognizable velocity of propagation." As general as this definition seems to be, it precludes light waves, which do not require a medium in which to propagate. Therefore, the definition used here will modify this definition slightly to read: a wave is any recognizable signal that is transferred from one position to another with a recognizable velocity of propagation. 
Whitham then proceeds to define two main classes of waves, which he refers to as hyperbolic and dispersive waves. The former he defines with reference to the form of equation from which the waves arise, and the latter he defines with reference to the type of solution, in the linear case, which the system admits. He points out that these systems are not exclusive, but there is some overlap between the two.

Within each class he first discusses the linear equation from which the class gets its name. For the first class, the rather familiar ideas of a hyperbolic equation and the corresponding characteristics for this equation arise. The ideas of a dispersion relation, phase velocity and group velocity arise from a discussion of linear dispersive waves. Then nonlinear processes within each class are examined. For hyperbolic systems this leads to the ideas of overlapping: characteristics, discontinuous solutions and, in the case of gasdynamics, shock waves. For dispersive systems the generalization to nonlinear cases leads to dispersion relations where the amplitude enters the relations. The analysis of nonlinear, dispersive waves is an area of very active research, and Whitham has been an important contributor to this area. In this subsection some of the basic ideas mentioned above will be discussed for linear systems; the present report cannot examine nonlinear waves.

\section{a. Hyperbolic Waves}

Waves for which the mathematical description satisfies hyperbolic partial differential equations are called hyperbolic waves [26]. To discuss hyperbolic equations, a few definitions must be given, and general considerations related to hyperbolic equations will be discussed. Then the specialization of these general considerations to the prototype hyperbolic equation, the wave equation, will be presented. The presentation of this section will follow that of reference [26], chapter 5 .

A quasilinear system of first order equations is a system linear in the first derivatives of the dependent variables. The coefficients in the quasilinear system may depend both upon the independent variables and upon the dependent variables (making the system nonlinear). These systems can be examined for $n$ dependent variables and also an arbitrary number of independent variables. However, the most useful system to analyze is one consisting of $n$ dependent and two independent variables, taken to be time $t$ and position $x$. For this system the general equations are

$$
A_{i j} \frac{\partial u_{j}}{\partial t}+a_{i j} \frac{\partial u_{j}}{\partial x}+b_{i}=0 \quad i=1,2, \ldots, n
$$

where the $u_{j}, j=1,2, \ldots, \mathrm{n}$ are the dependent variables and $A_{i j}, a_{i j}$ and $b_{i}$ will generally depend upon $u_{j}, x$, and $t$.

Characteristics for this system of equations are curves in the $x, t$-plane along which the directional derivatives of the dependent variables are related: that is, along a characteristic curve the partial differential equations (P.D.E.'s) reduce to ordinary differential equations. Linear combinations of the P.D.E.'s can be found

$$
l_{i}\left(A_{i j} \frac{\partial u_{j}}{\partial t}+a_{i j} \frac{\partial u_{j}}{\partial x}\right)+l_{i} b_{i}=0
$$

such that all derivatives are in the same (characteristic) direction. Then the equations can be written

$$
m_{j}\left(\beta \frac{\partial u_{j}}{\partial t}+\alpha \frac{\partial u_{j}}{\partial x}\right)+l_{i} b_{i}=0 .
$$

The condition for such $l_{i}$ to exist is that

$$
\left|A_{i j}-\frac{\beta}{\alpha} a_{i j}\right|=0
$$

for some ratio $\beta / \alpha$. The curves

for which

$$
t=T(\eta), x=X(\eta)
$$

$$
\frac{d T}{d \eta}=\beta, \frac{d X}{d \eta}=\alpha
$$


are the characteristics. Here, $\eta$ is a parameter which varies along the curve. The equation

$$
m_{j}\left(\frac{d T}{d \eta} \frac{\partial u_{j}}{\partial t}+\frac{d X}{d \eta} \frac{\partial u_{j}}{\partial x}\right)+l_{i} b_{i}=m_{j} \frac{d u_{j}}{d \eta}+l_{i} b_{i}=0
$$

is then said to be written in characteristic form. It is an ordinary differential equation relating variations of the dependent variables along the characteristic direction.

A definition of a hyperbolic P.D.E. can now be made. A system of equations (1), satisfying the condition that the matrices in the equations not be singular

$$
\left|\lambda A_{i j}+\mu a_{i j}\right| \neq 0
$$

for some $\lambda$ and $\mu$, is hyperbolic if $n$ linearly independent real vectors

$$
\mathbf{l}^{(k)}, k=1,2, \ldots, n
$$

can be found such that

$$
l_{i}^{(k)}\left(A_{i j} \alpha^{(k)}-a_{i j} \beta^{(k)}\right)=0
$$

for each $k$, and the corresponding directions $\left(\alpha^{(k)}, \beta^{(k)}\right)$ are real with

$$
\left(\alpha^{(k)}\right)^{2}+\left(\beta^{(k)}\right)^{2} \neq 0 .
$$

For a definition of hyperbolic systems of quasi-linear P.D.E. extended to $m$ independent variables, see reference [26].

As an illustrative example of a system of quasi-linear hyperbolic equations, consider the equations of gasdynamics for one-dimensional, unsteady, isentropic flow:

$$
\begin{aligned}
& \frac{\partial \rho}{\partial t}+u \frac{\partial \rho}{\partial x}+\rho \frac{\partial u}{\partial x}=0 \\
& \frac{\partial u}{\partial t}+u \frac{\partial u}{\partial x}+\frac{1}{\rho} \frac{\partial p}{\partial x}=0 \\
& \frac{\partial p}{\partial t}+u \frac{\partial p}{\partial x}-c^{2}\left(\frac{\partial \rho}{\partial t}+u \frac{\partial \rho}{\partial x}\right)=0 .
\end{aligned}
$$

Here $\rho$ is the gas density, $u$ its velocity and $p$ its pressure. $c^{2}$ is the speed of sound in the gas, defined by the relation

$$
c^{2}=\left(\frac{d p}{d \rho}\right)
$$

the derivative taken at constant entropy. The first equation is that of conservation of mass, the second is conservation of momentum and the third is conservation of entropy. The last equation is already in characteristic form. The first equation multiplied by $c^{2}$, the second by $\pm c \rho$ and the two added to the third equation produce the other two characteristic forms. Therefore,

$$
\begin{gathered}
\frac{\partial p}{\partial t}+(u+c) \frac{\partial p}{\partial x}+c \rho\left[\frac{\partial u}{\partial t}+(u+c) \frac{\partial u}{\partial x}\right]=0 \\
\frac{\partial p}{\partial t}+(u-c) \frac{\partial p}{\partial x}-c \rho\left[\frac{\partial u}{\partial t}+(u-c) \frac{\partial u}{\partial x}\right]=0 \\
\frac{\partial p}{\partial t}+u \frac{\partial p}{\partial x}-c^{2}\left(\frac{\partial \rho}{\partial t}+u \frac{\partial \rho}{\partial x}\right)=0
\end{gathered}
$$

or

along the characteristic defined by $\frac{d x}{d t}=u+c, \frac{d p}{d t}+c \rho \frac{d u}{d t}=0$ 
and

$$
\text { along the characteristic defined by } \frac{d x}{d t}=u-c, \frac{d p}{d t}-c \rho \frac{d u}{d t}=0
$$

$$
\text { along the characteristic defined by } \frac{d x}{d t}=u, \quad \frac{d p}{d t}-c^{2} \frac{d \rho}{d t}=0 \text {. }
$$

Hence, this set of equations is hyperbolic.

The equations of gasdynamics have formed the basis for much of the development of the theory of nonlinear hyperbolic equations. As can be seen, the characteristics are dependent upon the solution itself, and therefore the equations are nonlinear. The nonlinearity can lead the characteristics to overlap, and this "breaking" of compression waves produces shock waves. As pointed out already, the nonlinear aspects of the waves will not be considered here.

These equations, when linearized, lead to the wave equation, the classical hyperbolic equation describing waves. To linearize these equations, take as the undisturbed state a quiescent fluid $u_{0}=0$ with constant properties

$$
\rho_{0}, p_{0} \text { and } c_{0}^{2}=\left(\frac{d p}{d \rho}\right)_{0}
$$

Then let $\tilde{p}$, $\tilde{u}$, and $\tilde{\rho}$ represent the (small) deviation of the respective quantities from their undisturbed values. The equations can be written

$$
\begin{aligned}
& \frac{\partial \tilde{p}}{\partial t}+c_{0} \frac{\partial \tilde{p}}{\partial x}+c_{0} \rho_{0}\left(\frac{\partial \tilde{u}}{\partial t}+c_{0} \frac{\partial \tilde{u}}{\partial x}\right)=0 \\
& \frac{\partial \tilde{p}}{\partial t}-c_{0} \frac{\partial \tilde{p}}{\partial x}-c_{0} \rho_{0}\left(\frac{\partial \tilde{u}}{\partial t}-c_{0} \frac{\partial \tilde{u}}{\partial x}\right)=0
\end{aligned}
$$

The third equation becomes unnecessary because it simply relates density and pressure fluctuations through the sound speed. Let

$$
\left(\frac{\partial}{\partial t}-c_{0} \frac{\partial}{\partial x}\right)
$$

operate on eq (12),

$$
\left(\frac{\partial}{\partial t}+c_{0} \frac{\partial}{\partial x}\right)
$$

on eq (13), and add these relations. The result is the one-dimensional wave equation for the pressure fluctuation

$$
\left(\frac{\partial^{2}}{\partial t^{2}}-c_{0}^{2} \frac{\partial^{2}}{\partial x^{2}}\right) \tilde{p}=0
$$

The general solution for this equation is

$$
\tilde{p}=f\left(x-c_{0} t\right)+g\left(x+c_{0} t\right)
$$

and this solution describes small amplitude waves. The first function $f$ represents a wave which propagates unchanged in form to the right while the second function $g$ represents a wave propagating without distortion to the left. When the disturbance pressure and its time-derivative are known everywhere initially, these two functions are determined uniquely.

Equation (14) can also be written in factored form as

$$
\left(\frac{\partial}{\partial t}-c_{0} \frac{\partial}{\partial x}\right)\left(\frac{\partial}{\partial t}+c_{0} \frac{\partial}{\partial x}\right) \tilde{p}=0 .
$$


Either one of the factors represents the simplest linear hyperbolic equation, each factor giving rise to a wave solution. For example,

$$
\left(\frac{\partial}{\partial t}+c_{0} \frac{\partial}{\partial x}\right) \tilde{p}=0
$$

has as its general solution

$$
p=f\left(x-c_{0} t\right)
$$

A more general, quasi-linear form of this simple and important hyperbolic equation is [26]

$$
\left(\frac{\partial}{\partial t}+c(p) \frac{\partial}{\partial x}\right) p=0
$$

where the tilde has been dropped and $c(p)$ is a function of the dependent variable.

\section{b. Dispersive Waves}

Perhaps the more interesting and certainly the more challenging class of waves at this time is that of dispersive waves. For the linear case, a dispersive wave is recognized by its form of solution $\phi$, having the form

$$
\phi=A \exp (i \theta)
$$

for elementary solutions. Here $A$ is the amplitude and $\theta$ is the phase. In the simplest case $A$ is a constant and $\theta$ has the form

$$
\theta=\mathbf{k} \cdot \mathbf{x}-\omega t
$$

Here $\mathbf{k}$ is the wave number and $\omega$ is the frequency and each is constant. Since the equations are linear, the amplitude factors out and is arbitrary, while the frequency and wave numbers are related by the governing equations through the so-called dispersion relation

$$
G(\omega, \mathbf{k})=0
$$

The dispersion relation can be solved for the frequency as a function of wave number. In general there will be more than one function $\omega=W(\mathbf{k})$ which satisfies the dispersion relation; each such solution is called a mode of the system. Since the governing equations are linear, various modes of the system can be superposed.

When $\theta$ is taken to be constant, surfaces of constant phase are determined. In the simplest case, surfaces of constant phase are planes. The phase determines the position in the cycle between a crest, where the real part of $\phi$ is maximum, and a trough, where real $\phi$ is minimum. The normal distance between two surfaces of constant phase representing successive crests (or troughs) is the wave length $\lambda$ and is equal to

$$
\lambda=2 \pi /|\mathbf{k}| .
$$

The planes of constant phase are seen to change position, but not orientation as time increases; therefore, the surfaces of constant phase propagate as time evolves. At a fixed position the solution is seen to undulate as successive crests and troughs pass the point. The period $\tau$ between successive crests is equal to

$$
\tau=\frac{2 \pi}{\omega}
$$

The propagation velocity for the surfaces of constant phase is the phase velocity $\mathbf{c}$ for the wave and is equal to

where

$$
\mathbf{c}=\frac{\lambda}{\tau} \hat{k}=\frac{\omega}{|\mathbf{k}|} \hat{k}
$$

$$
\hat{k}=\frac{\mathbf{k}}{|\mathbf{k}|}
$$

is a unit vector in the $\mathbf{k}$ direction. 
The meaning of the term dispersion can now be seen. A wave of particular wave number $\mathbf{k}$ propagates at a specified phase velocity $\mathbf{c}$ determined by the frequency $\omega=W(\mathbf{k})$ and the wave number. For another wave of different wave number, the freuqnency and the phase velocity will, in general, be different. Hence the waves will disperse as time evolves. Witham [26] defines a dispersive wave to be one having the form (19) with phase (20) satisfying the dispersion relation (21) such that, if $\omega=W(\mathbf{k})$ is a mode, $W(\mathbf{k})$ is real and the determinant

$$
\left|\frac{\partial^{2} W}{\partial k_{i} \partial k_{j}}\right| \neq 0 \text {. }
$$

The last two conditions guarantee that the solution will in fact be wave-like and that the phase velocity will not be constant for all wave numbers (so that the waves do disperse).

A very important concept in the discussion of dispersive waves is the concept of group velocity. The group velocity is the velocity with which a "group" of waves, or a general wave composed of a distribution of elementary waves of different wave number, would propagate. This concept can be introduced in a variety of ways $[24,26]$. Here it will simply be noted that, in the one-dimensional case, when two waves of equal amplitude and wave numbers $k_{1}$ and $k_{2}$ and frequencies $\omega_{1}$ and $\omega_{2}$ respectively are superposed, their sum can be written

$$
\begin{aligned}
\phi & =A \cos \left(k_{1} x-\omega_{1} t\right)+A \cos \left(k_{2} x-\omega_{2} t\right) \\
& =2 A \cos \left(\frac{k_{1}+k_{2}}{2} x-\frac{\omega_{1}+\omega_{2}}{2} t\right) \cos \left(\frac{k_{2}-k_{1}}{2} x-\frac{\omega_{2}-\omega_{1}}{2} t\right) .
\end{aligned}
$$

In this latter form the superposition is seen to be the product of a wave with average wave number $\left(k_{1}+k_{2}\right) / 2$ and average frequency $\left(\omega_{1}+\omega_{2}\right) / 2$ and a relatively slowly varying, modulating wave with wave number $\left(k_{1}-k_{2}\right) / 2$ and frequency $\left(\omega_{2}-\omega_{1}\right) / 2$. A plot of this function for a fixed time shows that the amplitude of the high frequency or short wavelength wave (the first cosine term) is modulated by the low frequency wave over a wavelength $4 \pi /\left(k_{2}-k_{1}\right)$. The low frequency or modulating wave propagates with a velocity

$$
\frac{\omega_{2}-\omega_{1}}{k_{2}-k_{1}}
$$

which in the limit of $k_{2} \rightarrow k_{1}$ and $\omega_{2} \rightarrow \omega_{1}$, becomes the group velocity in the one-dimensional case

$$
c_{g}=\frac{d \omega}{d k}
$$

In the general three-dimensional case, the group velocity is defined by the equation

$$
\mathbf{c}_{g}=\nabla_{\mathbf{k}} \omega
$$

where $\nabla_{\mathbf{k}}$ means the gradient with respect to the components of the wave number vector $\mathbf{k}$. It can be shown that energy and several other features propagate with this velocity [26].

When the medium within which a wave propagates is anisotropic, waves which are launched in one direction propagate with a velocity different from those launched in a different direction. Then both the phase velocity and group velocity depend upon the direction in which the waves propagate. However, in a dispersive, anisotropic medium a very surprising phenomenon arises: generally the group velocity is in a different direction than the phase velocity. Therefore, in general, energy propagates in a direction different from that in which individual component waves propagate, leading to surprising and interesting results. A general mathematical formulation of these waves by Fourier analysis will be discussed in the next subsection.

Stratified fluids, rotating fluids and electrically conducting fluids in the presence of magnetic fields are all examples of anisotropic media with respect to wave propagation. In each case there is a direction specified by the constraint on the fluid, gravity, rotation and the magnetic field, and waves propagating along the direction of the constraint experience different forces than do those propagating transverse to the constraints. The features of waves in stratified or rotating fluids will be discussed in some detail in remaining subsections. 


\subsection{Methodology for Analysis of Waves in Dispersive, Anisotropic Media}

As mentioned above, both Lighthill and Whitman have been very instrumental in the development of general methodology for the analysis of waves in dispersive and anisotropic media. Some of the extensions of these methods make them also applicable to inhomogeneous media, and considered in the broadest context, the methodology can be applied to the examination of nonlinear waves [26]. Since this entire topic is very broad and still very active, a complete survey is not within the scope of this report. Rather an outline of some of the features of the various methods will be undertaken, and a more detailed presentation of the methods most familiar to the author will be presented. Only linear waves will be considered here.

Two basic approaches to the study of waves in anisotropic and dispersive media have been developed. The first approach has been called the study of the kinematics of waves [24, 26] and also the description of waves in terms of rays [4]. The approach is very powerful in its most general form when local properties of the waves are sought. When boundaries must be considered, it is not clear that the method can be applied as conveniently. It is this theory, when pushed to greater generality, which provides methodology for examination of nonlinear waves. An outline of the simpler aspects of this approach will be presented in this subsection.

The second approach is a Fourier analysis approach and has been called the method of modes by Turner [4]. It has been developed to a highly useful form by Lighthill [25] and by others. In this subsection the method of Fourier analysis, including extraction of relevent information by asymptotic evaluation, will be emphasized because this general methodology has been found so useful in applications. Following this subsection, some applications will be presented.

\section{a. Kinematics of Waves}

As pointed out by Lighthill [18] and discussed in great detail by Whitham [26], the method of the kinematics of waves "makes use of the duality between waves and particles, that is fundamental to quantum mechanics, and follows a wave packet through the system as if it were a particle." 'The equations for the waves are developed in Hamiltonian form, a HamiltonJacobi equation is found for the phase of the wave, a Lagrangian is found for the wave motion and a variational approach to the determination of the equation is developed. One advantage to this approach is that the P.D.E. for the waves can be cast in the form of ordinary differential equations (O.D.E.) which are much easier to solve computationally. A second advantage is that this approach provides a systematic methodology for attacking nonlinear problems.

In the one-dimensional case, a statement of the conservation of waves in differential form can be written as

$$
\frac{\partial k}{\partial t}+\frac{\partial \omega}{\partial x}=0 .
$$

This equation states that the time rate of change of the number of waves per unit length (the wave number) plus the gradient of the flux of waves (the frequency) out of an element equals zero. If, in addition, a dispersion relation is known for the wave system, which in general depends upon position and time as well as wave number, then

$$
\omega=W(k, x, t)
$$

and the continuity equation can be written as

$$
\frac{\partial k}{\partial t}+\left(\frac{\partial W}{\partial k}\right) \frac{\partial k}{\partial x}=\frac{-\partial W}{\partial x} .
$$

In this form the equation is hyperbolic with a characteristic defined by

and characteristic form

$$
\frac{d x}{d t}=\frac{\partial W}{\partial k}
$$

$$
\frac{d k}{d t}=-\frac{\partial W}{\partial x}
$$

These two ordinary differential equations, obtained by writing the kinematic conservation equation and the disperson relation in characteristic form, are simply the equations of motion for a particle written in Hamiltonian form. The following identifications can be made: $x$ is the position of the wave in one case and of the particle in the other; just as in quantum mechanics, 
the wave number $k$ is identified with momentum $p$ and the dispersion function $W$ is identified with the Hamiltonian $H$. Furthermore, the frequency $\omega$ of the wave packet serves the same role as the energy $E$ in Hamiltonian mechanics, so that

$$
\begin{aligned}
& \frac{d \omega}{d t}=\frac{d W(k, x, t)}{d t}=\frac{\partial W}{\partial k} \frac{d k}{d t}+\frac{\partial W}{\partial x} \frac{d x}{d t}+\frac{\partial W}{\partial t} \\
& \frac{d \omega}{d t}=-\frac{\partial W}{\partial k} \frac{\partial W}{\partial x}+\frac{\partial W}{\partial x} \frac{\partial W}{\partial k}+\frac{\partial W}{\partial t} \\
& \frac{d \omega}{d t}=\frac{\partial \omega}{\partial t} .
\end{aligned}
$$

From these equations it can be seen that the wave packet travels with the group velocity

$$
c_{g}=\frac{\partial W}{\partial k} .
$$

When the medium is homogeneous, the dispersion relation is independent of position and

$$
\frac{d k}{d t}=0
$$

Hence the wave number (momentum) is constant along a characteristic (trajectory). When the dispersion relation is time independent, then

$$
\frac{d \omega}{d t}=0
$$

and the frequency (energy) is constant along a characteristic.

One more identification between waves and Hamiltonian mechanics will be made. The frequency and the wave number can be defined as partial derivatives of the phase function $\theta$ as follows

$$
\omega=-\frac{\partial \theta}{\partial t}, \quad k=\frac{\partial \theta}{\partial x} \text {. }
$$

Here $\theta$ can then be identified with the action in mechanics. With these definitions, the dispersion relation becomes the Hamilton-Jacobi equation when $\omega$ and $k$ are eliminated:

becomes

$$
\omega=W(k, x, t)
$$

$$
\frac{\partial \theta}{\partial t}+W\left(\frac{\partial \theta}{\partial x}, x, t\right)=0
$$

All of these equations can be generalized in a straightforward fashion to the three-dimensional case, and these equations will simply be listed.

or, in component form

$$
\begin{gathered}
\frac{\partial \mathbf{k}}{\partial t}+\nabla_{\mathbf{x}} \omega=0 \\
\omega=W(\mathbf{k}, \mathbf{x}, t) \\
\frac{d \mathbf{x}}{d t}=\nabla_{\mathbf{k}} W, \frac{d \mathbf{k}}{d t}=-\nabla_{\mathbf{x}} W
\end{gathered}
$$

$$
\begin{gathered}
\frac{d x_{1}}{d t}=\frac{\partial W}{\partial k_{1}} \\
\frac{d k_{1}}{d t}=-\frac{\partial W}{\partial x_{1}}
\end{gathered}
$$




$$
\begin{gathered}
\frac{d x_{2}}{d t}=\frac{\partial W}{\partial k_{2}} \\
\frac{d k_{2}}{d t}=-\frac{\partial W}{\partial x_{2}} \\
\frac{d x_{3}}{d t}=\frac{\partial W}{\partial k_{3}} \\
\frac{d k_{3}}{d t}=-\frac{\partial W}{\partial k_{3}} \\
\omega=\frac{\partial \theta}{\partial t}, k=\nabla_{\mathbf{x}} \theta
\end{gathered}
$$

and

$$
\frac{\partial \theta}{\partial t}+W\left(\nabla_{\mathbf{x}} \theta, \mathbf{x}, t\right)=0
$$

In this form (O.D.E.), these equations are ideally suited for ray-tracing techniques using conputational methods.

Finally, as noted before, this formulation of waves can be extended by considering the Lagrangian for the system and using variational methods with the Lagrangian. This methodology is very well suited for examination of nonlinear waves. Since it is an area of active research and application and since the scope of this paper is limited, the interested reader is referred to reference [26] for the extensions.

\section{b. Fourier Analysis and Asymptotics}

In this subsection, the outline of a Fourier analysis oî a class of linear P.D.E.'s describing waves in anisotropic, dispersive media will be given. The analysis was presented in this form by Lighthill [25], although similar analyses, of somewhat less generality, have been presented elsewhere. The analysis, as presented, is appropriate only to infinite homogeneous media, but extensions to inhomogeneous media or media with boundaries will be mentioned at the end. Applications of this analysis will be discussed in the following subsections.

For a general infinite, homogeneous medium in an undisturbed state, a small disturbance is governed by a linear P.D.E. with constant coefficients, which may be written

$$
P\left(i \frac{\partial}{\partial t},-i \frac{\partial}{\partial x},-i \frac{\partial}{\partial y},-i \frac{\partial}{\partial z}\right) \phi=0
$$

where $P$ is a polynomial and $\phi$ is a dependent variable specifying the wave. A plane-wave solution

$$
\phi=\phi_{0} \exp \{i(-\omega t+\mathbf{k} \cdot \mathbf{x})\}
$$

can exist if the dispersion relation

is satisfied.

$$
P\left(\omega, k_{1}, k_{2}, k_{3}\right)=0
$$

When the disturbance is produced by a localized forcing region, then the right side of eq (42) must be replaced by a "forcing term." For this inhomogeneous form of the equation, it is necessary to superpose the various plane-wave modes for the homogeneous equation to obtain the solution. A very general form for such a forcing function is

$$
\exp \left(-i \omega_{0} t\right) f(\mathbf{x}-\mathbf{U} t)
$$

where $\omega_{0}$ is the frequency with which the forcing term oscillates and $\mathbf{U}$ is the velocity with which it travels. This forcing term represents one which translates and oscillates simultaneously exciting waves in the otherwise undisturbed medium. A slight modification to the functional form above allows one to consider transient phenomena as well as steadily time varying disturbances [25]. Here only forcing functions of the form above will be considered. 
By a localized forcing function it is meant that $f(\mathbf{x})=f(x, y, z)$ vanishes outside some region around the origin. Then it can be decomposed into a Fourier integral

$$
f(\mathbf{x})=\int_{-\infty}^{\infty} d k_{1} \int_{-\infty}^{\infty} d k_{2} \int_{-\infty}^{\infty} d k_{3} F(\mathbf{k})^{i \mathbf{k} \cdot \mathbf{x}}
$$

where $F(\mathbf{k})=F\left(k_{1}, k_{2}, k_{3}\right)$ is a regular function of $\mathbf{k}$. The equation

$$
P\left(i \frac{\partial}{\partial t},-i \frac{\partial}{\partial x},-i \frac{\partial}{\partial y},-i \frac{\partial}{\partial z}\right) \phi=e^{-i \omega_{0} t} f(\mathbf{x}-\mathbf{U} t)
$$

has the formal solution, using the Fourier-integral procedure,

$$
\phi=\int_{-\infty}^{\infty} d k_{1} \int_{-\infty}^{\infty} d k_{2} \int_{-\infty}^{\infty} d k_{3} \frac{F(\mathbf{k}) \exp \left\{i\left[-\omega_{0} t+\mathbf{k} \cdot(\mathbf{x}-\mathbf{U} t)\right]\right\}}{P\left(\omega_{0}+\mathbf{U} \cdot \mathbf{k}, \mathbf{k}\right)} .
$$

This solution to the inhomogeneous equation is not unique: any solution to the homogeneous equation can also be superposed to produce another solution. However, when the requirement is imposed that only outgoing waves appear at infinity, the so-called radiation condition, then the solution becomes unique. Lighthill $[17,25]$ has given a prescription for applying the radiation condition and making the solution unique. The denominator of the integrand in eq (48) is the dispersion relation for waves excited by the forcing function. Points at which this function vanishes are poles of the transform kernel. Using residue theory, one of the Fourier transforms can be inverted exactly, and the radiation condition can be applied during this inversion $[17,25]$.

The solution, after one inversion has been performed, is still very formidable. Under special circumstances the other two integrals can also be inverted in terms of known functions; however, such inversions are the exception rather than the rule. As a result, asymptotic evaluation of the solution in the far field is very important for examination of the wave system. Again, Lighthill [17, 25] has given a prescription for such asymptotic evaluation using a multidimensional (two-dimensional in this case) stationary-phase analysis.

Since $F(\mathbf{k})$ is a regular function of each $k_{i}, F(\mathbf{k})$ has no singularities and can be taken as a smooth, well-behaved function; therefore it remains passive in the asymptotic analysis. The key to the asymptotic analysis is the dispersion relation

$$
P\left(\omega_{0}+\mathbf{U} \cdot \mathbf{k}, \mathbf{k}\right)=0 .
$$

For fixed $\omega_{0}$ and $\mathbf{U}$, the dispersion relation describes a surface in wave number space. At regular points on this surface, points at which the curvature of the surface does not vanish, a twodimensional steepest-descents procedure can be applied. This procedure determines both the directions in real space into which waves spread and the rate of decay of such waves with distance from the forcing function.

When the curvature of the dispersion-relation surface vanishes, a more involved steepestdescent procedure must be used. In the directions in real space for which this occurs, the amplitude of the waves fall off more slowly with distance from the forcing function than in other directions. For details of the asymptotic procedure, references [17] and [25] should be consulted.

When inhomogeneous media (media whose properties depend upon position) or finite media (media with boundaries) are considered, the problems become more difficult and often recourse must be made to numerical methods. Under these conditions, the inhomogeneity or the boundaries can change the spectrum of the eigenvalues from continuous to discrete. In the next subsection on stratified-fluids, where boundaries and inhomogeneities are important, this will be discussed in more detail.

\subsection{Waves in Stratified Fluids}

In this subsection a survey of internal waves in stratified media will be presented. The emphasis will be upon waves in a quiescent, stratified fluid using a modal analysis, as discussed in the previous subsection. First, the equations will be derived. Then a discussion of results will be presented.

a. Derivation of the Linearized Equation for the Vertical Displacement

The equations for mass and momentum conservation are eqs (2.11) and (2.12). [Note that equations will be numbered consecutively in each section of this report. When equations from 
a preceding section are referenced, the equation number will be prefixed by the section number, as the equations referenced above.] Equation (2.11) will be written as

$$
\frac{D \rho}{D t}+\rho \nabla \cdot \mathbf{u}=\rho Q
$$

Here $Q$ is a source term; this term has been included to discuss the modeling of internal waves (IW) excited by body motions or by "wake-collapse." Equation (2.12) will be written in a nonrotating reference frame

$$
\frac{D \mathbf{u}}{D t}+\frac{1}{\rho} \nabla p-g \mathbf{i}_{z}=0
$$

where $\mathbf{g}=-g \mathbf{i}_{z}$ and $\mathbf{i}_{z}$ is a unit vector in the $z$ direction, which is taken to be positive upward.

The equation of energy conservation, eq (2.16), will be rewritten with the energy addition term set to zero. This isentropic relation then becomes

$$
\frac{\partial p}{\partial t}+\mathbf{u} \cdot \nabla p=c^{2}\left(\frac{\partial \rho}{\partial t}+\mathbf{u} \cdot \nabla \rho\right)
$$

For an incompressible fluid the speed of sound $c$ becomes infinite, or the density following a fluid element does not change. Hence

and from the continuity equation

$$
\frac{D \rho}{D t}=\frac{\partial \rho}{\partial t}+\mathbf{u} \cdot \nabla \rho=0
$$

$$
\nabla \cdot \mathbf{u}=Q .
$$

In equilibrium the fluid is quiescent and stratified in the vertical direction, so that

$$
\begin{gathered}
\mathbf{u}_{0}=\left(u_{0}, v_{0}, w_{0}\right)=0 \\
Q_{0}=0 \\
\rho_{0}=\rho_{0}(z) \\
p_{0}(z)-p_{0}(z=0)=-g \int_{0}^{z} \rho_{0}\left(z^{\prime}\right) d z^{\prime} .
\end{gathered}
$$

The disturbance quantities are denoted by primes,

$$
\begin{gathered}
\mathbf{u}=\left(u^{\prime}, v^{\prime}, w^{\prime}\right) \\
Q=Q^{\prime} \\
\rho=\rho_{0}(z)+\rho^{\prime} \\
p=p_{0}(z)+p^{\prime} .
\end{gathered}
$$

These quantities are substituted into eqs (51), (53), and (54); and the equations are linearized with respect to disturbance quantities. The prime notation on disturbance quantities is dropped, and diff erentiation is denoted by subscripts. Then eqs (54), (53), and (51) become

$$
\begin{gathered}
u_{x}+v_{y}+w_{z}=Q \\
\rho_{t}+w \rho_{0, z}=0 \\
u_{t}=-\frac{1}{\rho_{0}} p_{x}, v_{t}=-\frac{1}{\rho_{0}} p_{y}, w_{t}=-\frac{1}{\rho_{0}} p_{z}-\frac{g \rho}{\rho_{0}} .
\end{gathered}
$$


These equations can be reduced to a single equation for the vertical displacement $\zeta$ of a fluid element from its equilibrium position:

In this equation

$$
\left(\partial_{t}{ }^{2}+N^{2}\right)\left(\zeta_{x x}+\zeta_{y y}\right)+\zeta_{z z t t}=Q_{z t}+\frac{\rho_{0, z}}{\rho_{0}}\left(Q_{t}-\zeta_{z t t}\right)
$$

and

$$
\partial_{t} \equiv \frac{\partial}{\partial t}
$$

$$
N^{2}=-g\left(\rho_{0, z} / \rho_{0}\right)
$$

is the square of the Brunt-Väisälä frequency, which, in general, is a function of the depth $z$. The last term on the right is taken to be zero by the Boussinesq approximation; therefore the final equation for the vertical displacement is

$$
\left(\partial_{t}{ }^{2}+N^{2}\right)\left(\zeta_{x x}+\zeta_{y y}\right)+\zeta_{z z t t}=Q_{z t} .
$$

Equation (59) agrees with the linear portion of eq (5.24) in reference [5]. Some authors have used other dependent variables or pairs of equations to formulate problems in stratified fluids.

To complete the formulation of problems in stratified fluids, boundary conditions must be applied. If the fluid is considered to be infinite and the source of waves localized, then a radiation condition (only outward propagating waves) must be applied at infinity. If the fluid is bounded by a solid surface, then the velocity normal to the surface must vanish. If there is a free surface, then the pressure remains continuous across the free surface.

Finally, initial conditions must also be specified. Since eq (59) is second order in time, both the initial displacement field and the initial vertical velocity field can be specified. The initial conditions can be applied formally if Laplace transform techniques are utilized. If steady state waves only are of interest, the initial conditions and transient effects may be ignored.

\section{b. A Survey of Results on Internal Waves in a Quiescent Stratified Fluid}

In an incompressible fluid the stratification can vary in a variety of ways. The simplest case to analyze is that in which the Brunt-Väisälä frequency is constant and the fluid is infinite. (A constant Brunt-Väisälä frequency implies that the fluid is exponentially stratified with density

$$
\rho_{0}(z)=\rho_{00} \exp \left(-\frac{N^{2}}{g} z\right)
$$

where $\rho_{00}$ is the density at $z=0$ and $z$ is measured positive upward.) This case also illustrates many of the interesting phenomena which occur in stratified fluids. In this subsection a survey will be given of some of the books and papers which describe internal waves; this survey will include a summary of methods and results, but will not include mathematical detail.

As shown above, eq (59) governs the internal-wave motion in a Boussinesq fluid. For an infinite fluid the plane-wave solution will satisfy this equation:

$$
\zeta=A \exp \{i(-\omega t+\alpha x+\beta y+\gamma z)\} .
$$

(The notation has been changed from that used in subsection $3 \mathrm{~b} ; \alpha, \beta$, and $\gamma$ are used as wave number components rather than $k_{1}, k_{2}$, and $k_{3}$.) This plane wave solution satisfies eq (59) for nonzero $A$ if the dispersion relation is satisfied:

$$
\left(\alpha^{2}+\beta^{2}\right)\left(N^{2}-\omega^{2}\right)-\gamma^{2} \omega^{2}=0 .
$$

From this relation it can be seen that plane waves, with $\alpha, \beta$, and $\gamma$ all real, only exist provided that $\omega \leq N$. If $\omega>N$, one of the wave numbers must be imaginary for the dispersion relation to be satisfied, and internal waves cannot propagate. Therefore, $N$ is a high-frequency cutoff for internal waves.

If the wave numbers are written in spherical polar coordinates

$$
\alpha=k \sin \theta \cos \Phi, \beta=k \sin \theta \sin \Phi, \gamma=k \cos \theta
$$


where $k$ is the magnitude of the wave number and $\theta$ and $\Phi$ are the angles defining its direction, then the dispersion relation becomes

or

$$
\tan ^{2} \theta=\frac{\omega^{2}}{N^{2}-\omega^{2}}
$$

$$
\sin ^{2} \theta=\omega^{2} / N^{2}
$$

Therefore the frequency of the wave does not depend upon the magnitude of the wave number, but only upon its direction. The anisotropic nature of the medium can be clearly seen from this form of the dispersion relation: for a plane wave of fixed frequency the direction of the wave number with respect to the vertical axis in wave number space is fixed.

The phase velocity, defined in eq (24), is

$$
\mathbf{c}=\frac{\omega}{|k|^{2}} \mathbf{k}=\frac{\omega}{\left(\alpha^{2}+\beta^{2}+\gamma^{2}\right)}\left\{\begin{array}{l}
\alpha \\
\beta \\
\gamma
\end{array}\right\}
$$

and the group velocity, defined in eq $(29)$, is

$$
\mathbf{c}_{g}=\nabla_{k} \omega=\frac{1}{\gamma^{2}} \frac{\left(N^{2}-\omega^{2}\right)^{2}}{\omega^{2}}\left\{\begin{array}{c}
\alpha \\
\beta \\
-\frac{\left(\alpha^{2}+\beta^{2}\right)}{\gamma}
\end{array}\right\} .
$$

The dot product of $\mathbf{c}$ and $\mathbf{c}_{g}$ shows the rather unusual property that the phase velocity and the group velocity of these internal waves are orthogonal. Therefore, energy is propagated at right angles to the velocity of an individual wave. This property is a result of the fact that the medium is both anisotropic and dispersive.

Görtler [27] examined the steady-state wave configuration in a homogeneous medium of constant Brunt-Väisälä frequency. He discussed the properties of the wave system in terms of characteristics of the linearized equations of motion noting that $N$ is the high-frequency cutoff for internal waves. Using a Schlieren system, he determined the wave-configuration experimentally.

In an independent study, Mowbray and Rarity [28] examined the two-dimensional internal waves excited by a cylinder oscillating in a constant- $N$ stratified medium. In their experiments they duplicated the results of Görtler using a schlieren system and explained these results in terms of the energy propagated by the group velocity in the anisotropic, dispersive medium. They found that, when internal waves are excited, significant amplitudes occur only in regions emanating along preferred directions from the oscillating cylinder. These directions are determined by the angle $\theta$, measured from horizontal, where

$$
\sin \theta= \pm \omega / N
$$

For a specified frequency $\omega, \theta$ is the angle of the group velocity. The variation of the angle $\theta$ with variation of frequency $\omega$ was measured, and $N$ was verified as the high-frequency cutoff.

From the form of the plane-wave solution (60) and the dispersion relation (61), it is apparent that the eigenvalues of this system form a continuous spectrum. From the principle of superposition, an integral over the eigenfunctions (60) form the general solution to problems in an infinite, constant- $N$ medium, as discussed previously. When boundaries are present or the Brunt-Väisälä frequency varies with depth, the eigenvalues can change from a continuous to a discrete spectrum.

To illustrate this behavior, a layer of fluid of finite depth $D$ will be considered. In this case, the general solution can be written as a function of $z$ times a horizontal plane wave:

$$
\zeta=Z(z) \exp \{i(-\omega t+\alpha x+\beta y)\}
$$

and the homogeneous version of eq (59) becomes

$$
\omega^{2} Z^{\prime \prime}+\left[N^{2}(z)-\omega^{2}\right]\left(\alpha^{2}+\beta^{2}\right) Z=0
$$


where the prime denotes differentiation with respect to $z$. This equation corresponds to eq (5.2.8) in reference [5]. The boundary conditions for eq (68) are that the vertical velocity $\frac{\mathrm{d} \zeta}{d t}$ vanish at $z=-D$ and that the pressure remain constant (at atmospheric pressure) at the free surface $z=0$. Phillips [5] presents the proper boundary condition to first order at the free surface; but for internal waves this surface can be treated approximately as a rigid boundary [29]. Therefore, at $z=0$, the vertical velocity vanishes, and, in terms of the vertical displacement, $\zeta=Z=0$ at $z=0$ and at $z=-D$.

For general variations of $N(z)$, solutions can be found only for discrete values of the frequency $\omega_{n}$. Corresponding to each eigenvalue $\omega_{n}$ is an eigenfunction $\psi_{n}(z)$, and any solution to the inhomogeneous form of eq (68) can be expanded in terms of these discrete eigenfunctions. If $N$ is constant in the layer for example,

and

$$
\psi_{n}=\sin \frac{n \pi z}{D}
$$

$$
\frac{\omega_{n}}{N}=\left[\frac{\left(\frac{\pi n}{D}\right)^{2}+\alpha^{2}+\beta^{2}}{\alpha^{2}+\beta^{2}}\right]^{1 / 2}
$$

An inhomogeneous form of eq (68) arises when disturbances are produced by nonzero initial conditions on the displacement or its time derivative, or by some internal forcing function.

Several authors have examined waves in inhomogeneous, stratified media. Eckart [6] has discussed the general methods for analysis presented in earlier subsections: he has discussed both ray-tracing techniques and modal techniques for analyzing waves. Also, he has discussed the effects upon the wave systems of compressibility and of spherical geometry. Furthermore, he has considered inertial waves in rotating fluid and some of the aspects of the infinitesimal waves in rotating and stratified fluids. Reference 6 is the most complete examination of waves in stratified and/or rotating fluids with application to geophysical fluid dynamics. No effects of turbulence or viscosity are considered.

In a chapter on waves of small amplitude, Yih [7] has examined stratified, compressible fluids, concentrating on fluids of finite depth. He has examined several mathematical questions related to problems that arise in studies of these fluids.

Phillips [5] has discussed the internal waves in a finite layer of incompressible fluid when the variation of the Brunt-Väisälä frequency models a thermocline. He derives eq (68) and notes some of the properties of this equation for a peaked distribution of $N(z)$. Equation (68) is in a form such that Sturm-Liouville theory can be applied directly; the modes of this equation which can be excited depend upon the value of $\omega$ with respect to $N_{\max }$, the maximum value of the BruntVäisälä frequency. When $\omega>N_{\max }$, no internal waves are excited, and this is a statement that $N_{\max }$ is the high-frequency cutoff. When $\omega<N_{\max }$, discrete values of $\omega$ exist as eigenvalues for the problem. In the lowest of these modes for internal waves, $Z(z)$ has its maximum near the maximum of $N(z)$, that is, near the thermocline. In this mode the whole thermocline heaves up and down in phase. The second eigenfunction or mode undergoes one change of sign in the thermocline region. This mode represents an internal wave which thickens and thins the thermocline region. Each higher mode has one more change of sign with oscillatory behavior in between in the thermocline region.

Several other aspects of internal-wave phenomena will be discussed briefly. Excitation of internal waves, waves by a body traveling through a stratified fluid, has been an area of rather active applied research during the past few years. It has been found that there are three ways in which a moving body can excite internal waves in an incompressible fluid. First, because of the body motion, a fluid element directly in the path of the body will be displaced as the body passes, and this displacement will produce internal waves. This effect has been studied both theoretically and experimentally by many people.

A key study was performed by Miles [29] who calculated the magnitude of the internal waves generated by this effect in both a constant- $N$ model and a thin-thermocline model of the fluid. Important features of this analysis are that the internal waves are related to motion and the size of the body using a small, slender-body approximation and that a stationary-phase analysis is used to calculate the far-field properties. Carrier and Chen [30] analyzed this effect in the far field for a three-layer model for the thermocline structure and for a finite size body. Experimentally, the internal-wave phase configuration was observed first by Stevenson [31]. Additional work in this area has been concerned with refining theoretical models of the thermo- 
cline structure, examining the interaction of internal waves with a fluid surface and with making measurements of internal-wave amplitudes.

A second mechanism by which a moving body may produce internal waves is by "wakecollapse" [32]. Any body moving through a fluid produces a turbulent wake due to frictional drag and, if it propels itself, due to the propellor action. The turbulent wake grows with distance behind the body, mixing the ambient fluid into the wake and tending to homogenize the fluid. At a certain distance downstream from the body, growth of the wake ceases, and, due to the buoyancy effects, the wake begins to collapse. During collapse, the fluid in the wake moves to the level at which its density is equal to the ambient density. The collapse is a reasonably efficient generator of internal waves.

Studies of wake collapse and internal-wave generation have proceeded along two lines. One line of study is the examination of the turbulent-wake properties and scaling of these properties with body size, speed etc. [33, 34, 4].

The other line of study has emphasized the internal waves produced by such collapse $[35,29,30]$. Most of the work along both lines has appeared in report form only; the references given here are indicative of the type of studies being carried out.

A third mechanism by which internal waves may be excited by a body in a stratified fluid is through oscillations of the body at a frequency below the Brunt-Väisälä frequency [28]. When the body remains at a fixed location and bobs up and down or "breathes fluid" in and out, these waves have been analyzed [36, 37] and measured experimentally [38]. When the body is both translating and oscillating at a prescribed frequency, either greater or less than the Brunt-Väisä]ä frequency, the internal-wave system has been analyzed [39] and the phase configuration of the waves have been observed experimentally [40].

Limitations of space do not allow any discussion of other currently interesting areas of research concerning small-amplitude internal waves. The books of Turner [4] and Phillips [5] discuss some of these, for example, internal waves in moving stratified media and weak nonlinear interactions between waves.

\subsection{Waves in Rotating Fluids}

In this subsection, a survey of inertial waves will be presented. It will be rather brief because many of the methods for analysis of the waves were discussed before and because many of the results are the same as those in stratified fluids. First, the equations governing smallamplitude waves in rotating fluid will be derived, and features of intertial waves in an unconfined, uniformly rotating fluid will be presented. Then a short discussion of Rossby waves, which arise in more general rotating flows, will be given.

\section{a. Derivation of Equations}

From the equations for mass and momentum conservation, eqs (2.11) and (2.12), the basic equation for the vorticity of a fluid element can be derived [9, 10]. Formally, the vorticity is defined as the curl of the velocity vector, and physically it is twice the angular velocity of rotation of a fluid element. Equation (2.12) can be rewritten as

$$
\frac{\partial \mathbf{u}}{\partial t}+\frac{1}{2} \nabla u^{2}-\mathbf{u} \times(\boldsymbol{\omega}+2 \boldsymbol{\Omega})=-\frac{1}{\rho} \nabla p+\nabla\left[-g z+\frac{1}{2}(\boldsymbol{\Omega} \times \mathbf{r}) \cdot(\boldsymbol{\Omega} \times \mathbf{r})\right]
$$

When the curl of this equation is taken, an equation for the vorticity $\omega \equiv \nabla \times \mathbf{u}$ results

Using eq (2.11), assuming.

$$
\frac{\partial \omega}{\partial t}-\nabla \times[\mathbf{u} \times(\boldsymbol{\omega}+2 \boldsymbol{\Omega})]=-\nabla\left(\frac{1}{\rho}\right) \times \nabla p .
$$

$$
\frac{\partial \Omega}{\partial t}=0
$$

and manipulating somewhat, eq (70) can be rewritten as

$$
\frac{D}{D t}\left(\frac{\omega+2 \Omega}{\rho}\right)=\frac{\omega+2 \Omega}{\rho} \cdot \nabla \mathbf{u}-\frac{1}{\rho} \nabla\left(\frac{1}{\rho}\right) \times \nabla p .
$$

Equation (71) is an equation for the absolute vorticity $[9,10], \boldsymbol{\omega}+2 \boldsymbol{\Omega}$, which is the vorticity in an inertial (nonrotating) reference frame. It states that the time rate of change of the absolute vorticity divided by the density following a fluid element is influenced by two processes. The 
first is simply the change in vorticity due to deformation of the vortex lines (stretching and tilting) as the vortex lines are convected about with the fluid (see ref. [9] and references therein concerning vorticity). The second term is a production term for vorticity resulting when the gradient of the density and temperature are not parallel. When these gradients are parallel, this term is zero, and pressure is a function of density. (When viscous effects are also important, another term is present on the right-hand side of eq (71), and this term is diffusive in nature. Several standard references on fluid dynamics have discussions of the viscous diffusion of vorticity).

The vorticity is a very useful concept, and eq (71) is an important equation from which many useful relations can be derived. For example, the Taylor-Proudman theorem, (Eq 2.13a), follows directly from this equation when the flow is incompressible (or barotropic so that pressure is a function only of density), inviscid, steady and slow (so that products of vorticity $\boldsymbol{\omega}$ and velocity $\boldsymbol{u}$ can be neglected).

Equation (71) can also be used to derive other governing relations, such as the fundamental equation for small-amplitude, inviscid inertial waves in an unbounded, incompressible, rotating fluid. In an incompressible fluid $\nabla \cdot \boldsymbol{u}=0$, and, therefore, by the continuity equation (2.11),

$$
\frac{D \rho}{D t}=0
$$

Then, formally the density can be removed from within the substantial derivative in eq (71). Since $\Omega$ is constant, its substantial derivative is zero, and because nonlinear terms are being neglected, the substantial derivative of the vorticity reduces to the partial of this quantity with respect to time. On the right-hand side, the last term is zero because the fluid is assumed to be barotropic, and in the first term the nonlinear portion $\boldsymbol{\omega} \cdot \boldsymbol{\nabla} \boldsymbol{u}$ is neglected. Therefore, eq (71) becomes

$$
\frac{\partial \omega}{\partial t}=2 \Omega \cdot \nabla \mathbf{u}
$$

Taking the curl of eq (72) and eliminating the vorticity between the new equation and eq (72) yields a single for the velocity

$$
\left[\frac{\partial^{2}}{\partial t^{2}} \nabla^{2}+(2 \Omega \cdot \nabla)^{2}\right] \mathbf{u}=0
$$

This equation is the fundamental one for inertial waves in an inviscid, incompressible, uniformly rotating fluid [10]. As in the preceding subsection, an excitation source could have been included in this derivation, resulting in an inhomogeneous form for the equation. However, discussion will be restricted to the homogeneous form. The formulation is complete when initial and boundary conditions are specified. For an infinite fluid and steady-state considerations, only a radiation condition needs to be applied at infinity.

Another important and interesting equation can be derived from eq (71), an equation which governs the vertical component of vorticity in a rotating layer of incompressible fluid. In this approximation two key ideas are utilized. First since the fluid is rotating, the absolute vorticity obeys eq (71) (with the second term on the right-hand side zero) and the motion is essentially two dimensional, as the Taylor-Proudman theorem states when the motion is slow and steady. If $\boldsymbol{\Omega}=\Omega \hat{\mathbf{k}}$ where $\hat{\mathbf{k}}$ is a unit vector in the positive $z$ direction, then the motion is such that the fluid variables approximately only depend on $x$ and $y$. Then columns of fluid move about as units such that the horizontal components of velocity are uniform with height.

Second, since the fluid is incompressible, changes in the horizontal divergence of the fluid are accompanied by changes in the fluid height. Then the basic unit to consider to describe the fluid motion is the fluid column. If $H(x, y, t)$ is this height of the fluid, then the incompressibility condition $\nabla \cdot \mathbf{u}=0$ becomes

$$
-\left(\frac{\partial u}{\partial x}+\frac{\partial v}{\partial y}\right)=\frac{\partial w}{\partial z}=\frac{1}{H} \frac{D H}{D t}
$$

where 
is now the two-dimensional substantial derivative

$$
\frac{D}{D t}=\frac{\partial}{\partial t}+u \frac{\partial}{\partial x}+v \frac{\partial}{\partial y}
$$

With $\zeta=\omega \cdot \hat{k}$, the vertical component of eq $(71)$ becomes

$$
\frac{D}{D t}(\zeta+2 \Omega)=(\zeta+2 \Omega) \frac{\partial w}{\partial z}
$$

where, again, the substantial derivative is two dimensional since, as discussed above, $u$ and $v$ have been assumed to remain approximately constant with height.

Combination of eqs (74) and (75) yields

$$
\frac{D}{D t}\left(\frac{\zeta+2 \Omega}{H}\right)=0
$$

The quantity $(\zeta+2 \Omega) / H$ is called the potential vorticity $[9,10,73,74]$, and the relation $(76)$ is known as the conservation of potential vorticity. It states that the $z$ component of the absolute vorticity divided by the fluid layer thickness is constant following a fluid element (column). Its applicability to the fluid layers on a spherical surface for representing the oceans or atmosphere, is a topic of considerable interest $[9,10]$.

In the next subsection a brief discussion of inertial waves, starting from eq (73), will be presented. Then in the following subsection, eq (76) will be examined to show how another type of wave, Rossby waves, arise.

b. A Survey of Results on Inertial Waves in Unconfined Uniformly Rotating Fluids

For an infinite fluid the plane wave solution

$$
\mathbf{u}=\mathbf{V} \exp [i(-\omega t+\alpha x+\beta y+\gamma z)]
$$

will satisfy eq (73) provided that the dispersion relation

where

$$
\omega^{2} k^{2}-4 \Omega^{2} \gamma^{2}=0
$$

$$
k=\left(\alpha^{2}+\beta^{2}+\gamma^{2}\right)^{1 / 2}
$$

is the magnitude of the wave number. Equation (78) shows that solutions exist for real $\alpha, \beta$, and $\gamma$ only for $\omega \leq 2 \Omega$, exactly as in the stratified-fluid case with $N$ replaced by $2 \Omega$. If spherical polar coordinates are used in wave number space, then $\gamma=k \cos \theta$ and

$$
\cos ^{2} \theta=\frac{\omega^{2}}{4 \Omega^{2}}
$$

Comparison of eqs (79) and (63) shows that the dispersion relations are the same with $2 \Omega$ replaced by $N$ and $\cos \theta$ replaced by $\sin \theta$. Therefore, the observations made about internal waves apply also to inertial waves. For example, the phase velocity is

and the group velocity is

$$
\mathbf{c}=\frac{\omega}{\left(\alpha^{2}+\beta^{2}+\gamma^{2}\right)}\left\{\begin{array}{l}
\alpha \\
\beta \\
\gamma
\end{array}\right\}
$$

$$
\mathbf{c}_{g}=\nabla_{\mathbf{k}} \omega=\frac{2}{\omega} \frac{4 \Omega^{2} \gamma^{2}}{\left(\alpha^{2}+\beta^{2}+\gamma^{2}\right)^{2}}\left\{\begin{array}{c}
-\alpha \\
-\beta \\
\left(\alpha^{2}+\beta^{2}\right) / \gamma
\end{array}\right\}
$$

Therefore, as in the case of internal waves, the group velocity is orthogonal to the phase velocity for inertial waves.

The first discussions of the steady wave system in a rotating fluid were carried out by Görtler [41], who noted that the characteristic directions for the waves are given by the relation

$$
\cos \theta= \pm \omega / 2 \Omega
$$


where $\theta$ is the angle in physical space measured from horizontal. Subsequent analysis of the steady-state inertial waves excited by an oscillating disk in a rotating fluid were made by Oser [42], who then verified the analysis experimentally [43]. In his analysis Oser was able to express the solution for the small-amplitude waves in terms of known functions and to calculate the flow field near the disk as well as in the far field.

For a fixed rotational frequency $\Omega$, the oscillation frequency $\omega$ determines the direction of energy propagation for the inertial waves, eq (82). When the oscillation frequency is small, this direction becomes nearly vertical, whereas when $\omega \rightarrow 2 \Omega$, the direction is nearly horizontal. This behavior is to be contrasted with that in a stratified fluid. There the characteristic directions become nearly horizontal for small $\omega$ and nearly vertical as $\omega \rightarrow N$.

No additional studies on waves in rotating fluids will be discussed here due to space limitations. Some additional considerations of inertial waves in unbounded media are given by Greenspan [9], who, discusses, among other questions, the reflection of plane inertial waves by an interior surface, the slow transient motion of a disk along the axis of rotation (and how the phenomenon known as a Taylor column develops) and waves produced by a travelling forcing function (along the lines presented earlier for a stratified medium). Eckart [6] also presents analyses and results on waves in rotating fluids. Furthermore, he discusses smallamplitude waves in fluids when rotation and stratification are both present. Some more recent studies of waves in rotating and stratified fluids have been performed by Hendershott [36], who examined waves excited by an oscillating body, and by Rao [44], who examined waves excited by travelling forcing effects.

\section{c. Rossby Waves}

There exists in large scale geophysical flows another type of wave, the Rossby wave, which owes its existence to a mechanism other than that described in the last subsection. Here an attempt will be made to explain this mechanism, and some of the features of these waves will be discussed. More detailed discussions of Rossby waves, with consideration of laboratory experiments and of oceanographic and atmospheric waves, are given in references [9, 10].

The conservation of potential vorticity, eq (76), is a statement that the component of the absolute vorticity in the direction of the rotation vector divided by the depth of a fluid column is constant to an observer following the column. On the earth, the direction of the rotation vector is from the south to north poles so that the conservation of potential vorticity is strictly valid only in this direction. Often the conservation relation is applied for the component of absolute vorticity in the radial direction at that latitude. When this is done, $2 \Omega$ in eq (76) is replaced by $f=2 \Omega \sin \theta$, where $\theta$ is latitude, and $f$ may be either considered to be locally constant or to vary with latitude. The conditions under which the conservation of vorticity applies in the radial direction have been discussed in reference [9] for example.

Rossby waves can arise when either $f$ or $H$ varies with lateral position. For example, for relatively large scales on the earth, $f$ varies approximately linearly with latitude; in the northern hemisphere, $f$ will increase in the northerly direction (as $\theta$ increases):

$$
f \cong f_{0}+\beta y
$$

where $y$ is taken to increase to the north and $x$ to increase to the east. Substitution into eq (76) with $H$ constant, and linearizing gives

$$
\frac{\partial \zeta}{\partial t}+\beta v=0
$$

A similar situation occurs when the imposed angular velocity $2 \Omega$ is constant and the depth of the layer varies linearly with external position [9]. Again, eq (84) is approximately the governing equation, where $\beta$ is now a measure of this linear variation.

Equation (84) is simply a linearized statement of the conservation of potential vorticity. The variation with lateral position of the imposed angular velocity, eq (83), provides a restoring mechanism, and it is this restoring mechanism that gives rise to Rossby waves. For any disturbance, the rate of change of position in the northerly direction, $v$, produces a corresponding reduction of the time rate of change of its vorticity because of the variation in the imposed angular velocity $f$.

Equation (84) can be rewritten in terms of a single dependent variable, the stream function $\psi$; where

$$
u \equiv-\frac{\partial \psi}{\partial y}, v \equiv \frac{\partial \psi}{\partial x} .
$$

215-653 O - $77-4$ 
Then

$$
\zeta=\frac{\partial v}{\partial x}-\frac{\partial u}{\partial y}=\nabla^{2} \psi
$$

and eq (84) becomes

$$
\frac{\partial}{\partial t} \nabla^{2} \psi+\beta \frac{\partial \psi}{\partial x}=0
$$

a form examined in some detail by Lighthill, using Fourier analysis and asymptotic techniques.

\section{The Analogy Between Stratified and Rotating Fluids}

As discussed in the introduction, there is an analogy between stratified and rotating flows. Stratification and rotation provide constraints on the fluid flow, the stratification inhibiting the flow in the vertical direction and the rotation inhibiting flow in horizontal planes. In section 3 on Waves, this analogy was given mathematical form. There it was shown that internal waves in a stratified fluid of constant BV frequency behave like inertial waves in a rotating fluid.

In this section the mathematical analogy demonstrated in the previous section is extended. The review article of Veronis [16] on this analogy is used as the basis for this section. Since that article is both complete and clear, this section will be relatively brief and will concentrate only on the derivation of the equations from which the mathematical analogy is identified.

The motivation for examination of this analogy is provided by the desire to have a deeper understanding of both physical phenomena. Understanding phenomena in one system can clarify the analogous phenomena in the other system. In addition, laboratory studies in one system may be much more easily carried out than in the other system, allowing problems to be studied in the simpler system.

The systems considered will be assumed to have the axes of rotation and stratification coincident in the vertical direction. Dissipative effects such as viscosity and thermal conductivity will be included in the analyses. Also, boundaries are considered, but only those which are parallel or perpendicular to the rotational and gravitational direction. The analogy will also be seen to hold for systems which are both rotating and stratified.

In section 3, it was noted that waves in stratified and rotating fluids are related when the identification between the Brunt-Väisälä frequency $N$ and twice the rotational frequency $2 \Omega$ is made. Closer examination of the perturbation equations shows the further identifications which must be made in analogous flows. Equations (3.57) are the perturbation equations for waves in a stratified fluid. If it is assumed that there is no dependence of the waves upon spatial variables and that no sources are present, $Q=0$, then these equations become

$$
\begin{gathered}
\rho_{t}=-w \rho_{0, z} \\
u_{t}=v_{t}=0 \\
\rho_{0} w_{t}=-g \rho .
\end{gathered}
$$

Here, the continuity equation is identically satisfied, $\rho_{0}(z)$ is the equilibrium density distribution, $\rho$ is the perturbation density and $u, v$, and $w$ are the perturbation velocity components. From these equations, the velocities $u$ and $v$ in the horizontal direction are seen to be constant and taken to be zero. Only the remaining two equations for the density perturbation and the vertical velocity perturbation are relevant:

$$
\begin{gathered}
\rho_{0} \frac{\partial w}{\partial t}=-g \rho \\
\frac{\partial \rho}{\partial t}=-\rho_{0, z} w .
\end{gathered}
$$

The analogous perturbation equations can be obtained for a rotating fluid. If it is again assumed that there is no dependence upon spatial coordinates, then in component form these equations are

$$
\frac{\partial u}{\partial t}=2 \Omega v
$$




$$
\begin{gathered}
\frac{\partial v}{\partial t}=-2 \Omega u \\
\frac{\partial w}{\partial t}=0 .
\end{gathered}
$$

The last equation implies that the vertical velocity is constant, and it is taken to be zero. Only the first two equations are relevant.

Equations (2) and the first two of eqs (3) have exactly the same form; in fact, they can be made exactly the same if, in eqs $(2), u$ is substituted for $w, v$ is substituted for $\left(-g \rho / \rho_{0} N\right)$ and $2 \Omega$ is substituted for $N$. From eqs (2) and (3) and other simple examples of this nature, the following identifications, except for multiplicative factors, can be made [16]:

$\begin{array}{lrlllll}\text { Rotating } & \operatorname{system}(\Omega) & v & w & u & z & x \\ \text { Stratified system }(S) & -\rho & u & w & x & z .\end{array}$

The more general equations expressing the analogy for fluids which are both stratified and rotating will be derived following reference 16 . It is assumed that the stratified fluid can be described in the Boussinesq approximation, and other restrictions stated above also apply. The equation for the conservation of mass (2.11) becomes

$$
\nabla \cdot \mathbf{u}=0 .
$$

The equation for the conservation of momentum (2.12) becomes

$$
\frac{\partial \mathbf{u}}{\partial t}+\mathbf{u} \cdot \nabla \mathbf{u}+2 \Omega \times \mathbf{u}=-\frac{1}{\rho_{0}} \nabla P+\alpha g T \mathbf{i}_{z}+\nu \nabla^{2} \mathbf{u} .
$$

Here $P$ is the reduced pressure (including the centrifugal-force term and the static part of the gravitational-force term $[9,16])$. The buoyancy term is taken in a form appropriate for a Boussinesq fluid: the density is taken to be a linear function of the temperature variation $T$ from a reference value (see eq (2.24) and the discussion of that equation). The force $\mathbf{f}$ in eq (2.12) is taken to be the viscous force $\nu \nabla^{2} \mathbf{u}$.

The equation for the conservation of energy is eq (2.19). If the temperature is written as $\bar{T}+T$, where $\bar{T}(z)$ is the temperature distribution in the absence of motions, then eq $(2.19)$ becomes

$$
\frac{\partial T}{\partial t}+\mathbf{u} \cdot \nabla T+w \frac{d \bar{T}}{d z}=k \nabla^{2} T .
$$

Here it has been assumed that $\bar{T}(z)$ is a linear function of depth and that $T$ is the perturbation temperature caused by the motion. Equation (5) already implies this separation of the temperature. The constant temperature gradient will be defined to be equal to

$$
\frac{d \bar{T}}{d z}=4 \frac{\Delta T}{L}
$$

$\Delta T$ is the magnitude of the temperature difference over the vertical length scale $L$, the factor 4 being included for convenience. Then eq (6) becomes

$$
\frac{\partial T}{\partial t}+\mathbf{u} \cdot \nabla T+4 \frac{\Delta T}{L} w=k \nabla^{2} T .
$$

Equations (4), (5), and (7) will be nondimensionalized in two ways, depending upon the physical system to be modeled. For the first system, denoted $S_{\Omega}$, stratification is dominant with rotational effects modifying the motion. For the second system, denoted $\Omega_{S}$, rotation dominates and stratification modifies the basic motion.

A general nondimensionalization for both systems can be made using the following scales and nondimensional variables:

$$
\mathbf{u}=V \mathbf{u}^{\prime}, \quad t=\tau t^{\prime}, \quad \mathbf{r}=L \mathbf{r}^{\prime}, \quad P=\tilde{P} P^{\prime}, \quad T=2 \theta T^{\prime} .
$$


Here, the primed variables are dimensionless, and $V, \tau, L, \tilde{P}$ and $\theta$ are the dimensional scaling factors. Equations (4), (5), and (7) become

$$
\begin{gathered}
\nabla^{\prime} \cdot \mathbf{u}^{\prime}=0 \\
\frac{V}{\tau} \frac{\partial \mathbf{u}^{\prime}}{\partial t^{\prime}}+\frac{V^{2}}{L} \mathbf{u}^{\prime} \cdot \nabla^{\prime} \mathbf{u}^{\prime}+2 \Omega V \mathbf{i}_{z} \times \mathbf{u}^{\prime}=-\frac{\tilde{P}}{\rho_{0} L} \nabla^{\prime} P^{\prime}+2 g \alpha \theta T^{\prime} \mathbf{i}_{z}+\frac{\nu V}{L^{2}} \nabla^{\prime 2} \mathbf{u}^{\prime} \\
\frac{2 \theta}{\tau} \frac{\partial T^{\prime}}{\partial t^{\prime}}+\frac{2 \theta V}{L} \mathbf{u}^{\prime} \cdot \nabla^{\prime} T^{\prime}+\frac{4 \Delta T V}{L} w^{\prime}=\frac{2 k \theta}{L^{2}} \Delta^{\prime 2} T^{\prime} .
\end{gathered}
$$

\subsection{The Stratification-Dominated System, $S_{\Omega}$}

When stratification dominates, the various dimensional scaling factors are related by equating the magnitude of the time acceleration term, the pressure term and the buoyancy term in eq (9) Therefore,

$$
\frac{V}{\tau}=\frac{\tilde{P}}{\rho_{0} L}=g \alpha \theta
$$

Also, the magnitude of the time derivative of the temperature and the convective temperature changes due to the equilibrium temperature distribution are equated in eq (10). Then

$$
\frac{2 \theta}{\tau}=\frac{2 \Delta T V}{L}
$$

Equations (11) and (12) imply the following relations between scaling factors:

$$
\begin{gathered}
\tau=\left(\frac{L}{g \alpha \Delta T}\right)^{1 / 2}, \quad V=\left(\frac{L g \alpha}{\Delta T}\right)^{1 / 2} \theta \\
\theta=\frac{V \tau}{L} \Delta T, \quad \tilde{P}=\rho_{0} L g \alpha \theta .
\end{gathered}
$$

Equations (8)-(10) become, after dropping the prime notation,

$$
\begin{gathered}
\nabla \cdot \mathbf{u}=0 \\
\frac{\partial \mathbf{u}}{\partial t}+\epsilon \mathbf{u} \cdot \nabla \mathbf{u}+\frac{2}{F} \mathbf{i}_{z} \times \mathbf{u}=-\nabla P+2 T \mathbf{i}_{z}+\sigma^{1 / 2} R \nabla^{2} \mathbf{u} \\
\frac{\partial T}{\partial t}+\epsilon \mathbf{u} \cdot \nabla T+2 w=\sigma^{-1 / 2} R \nabla^{2} T
\end{gathered}
$$

where

$$
\begin{gathered}
\epsilon=\frac{V \tau}{L}=\frac{\theta}{\Delta T}, \quad F=\frac{1}{\Omega \tau} \\
\sigma=\nu / k=\text { the Prand tl number } \\
R=\frac{(\nu k)^{1 / 2} \tau}{L^{2}}=\left(\frac{\nu k}{g \alpha \Delta T L^{3}}\right)^{1 / 2}
\end{gathered}
$$

In the two-dimensional case, when all variation with respect to $y$ vanishes, these equations can be written in component form as (rearranging somewhat)

$$
\begin{gathered}
\frac{\partial w}{\partial t}+\epsilon \mathbf{u} \cdot \nabla w-2 T=-\frac{\partial P}{\partial z}+\sigma^{1 / 2} R \nabla^{2} w \\
\frac{\partial T}{\partial t}+\epsilon \mathbf{u} \cdot \nabla T+2 w=\sigma^{-1 / 2} R \nabla^{2} T
\end{gathered}
$$




$$
\begin{gathered}
\frac{\partial u}{\partial t}+\epsilon \mathbf{u} \cdot \nabla u-\frac{2 v}{F}=-\frac{\partial P}{\partial x}+\sigma^{1 / 2} R \nabla^{2} u \\
\frac{\partial v}{\partial t}+\epsilon \mathbf{u} \cdot \nabla v+\frac{2 u}{F}=\sigma^{1 / 2} R \nabla^{2} v \\
\frac{\partial w}{\partial z}+\frac{\partial u}{\partial x}=0 .
\end{gathered}
$$

\subsection{The Rotation-Dominated System, $\Omega_{S}$}

When rotation dominates, the various dimensional scaling factors are related by equating the magnitude of the time acceleration term, the Coriolis acceleration and the pressure term in eq (9). Then

$$
\frac{V}{\tau}=\Omega V=\frac{\tilde{P}}{\rho_{0} L}
$$

and these equations imply the following relations between the scaling factors:

$$
\tau=1 / \Omega, \quad \tilde{P}=\rho_{0} L V \Omega .
$$

Then eq (9) can be written, dropping the prime notation.

where

$$
\frac{\partial \mathbf{u}}{\partial t}+\epsilon \mathbf{u} \cdot \nabla \mathbf{u}+2 \mathbf{i}_{z} \times \mathbf{u}=-\nabla P+\frac{2 g \alpha \theta}{V \Omega} T \mathbf{i}_{z}+E \nabla^{2} \mathbf{u}
$$

$$
\begin{gathered}
\epsilon=\frac{V}{L \Omega}=\text { the Rossby number } \\
E=\frac{\nu}{\Omega L^{2}}=\text { the Ekman number. }
\end{gathered}
$$

Equation (10) can be written

$$
\frac{\partial T}{\partial t}+\epsilon \mathbf{u} \cdot \nabla T+\frac{2 \Delta T V}{\theta \Omega L} w=\frac{E}{\sigma} \nabla^{2} T .
$$

The magnitudes of the buoyancy term in eq (19) and of the term expressing convective temperature changes due to the equilibrium temperature distribution in eq (20) are also equated.

$$
\frac{2 g \alpha \theta}{V \Omega}=\frac{2 \Delta T V}{\theta \Omega L}
$$

This equation yields the relation between scaling factors

$$
\frac{V}{\theta}=\left(\frac{g \alpha L}{\Delta T}\right)^{1 / 2}
$$

Then eqs (19) and (21) can be written

where

$$
\begin{gathered}
\frac{\partial \mathbf{u}}{\partial t}+\epsilon \mathbf{u} \cdot \nabla \mathbf{u}+2 \mathbf{i}_{z} \times \mathbf{u}=-\nabla P+2 F T \mathbf{i}_{z}+\sigma^{1 / 2} \tilde{E} \nabla^{2} \mathbf{u} \\
\frac{\partial T}{\partial t}+\epsilon \mathbf{u} \cdot \nabla T+2 F w=\tilde{E} \sigma^{-1 / 2} \nabla^{2} T .
\end{gathered}
$$

$$
\begin{gathered}
F=\frac{1}{\Omega}\left(\frac{g_{\alpha} \Delta T}{L}\right)^{1 / 2}=\frac{N}{2 \Omega} \\
\tilde{E}=E \sigma^{-1 / 2}
\end{gathered}
$$




$$
N=\left(\frac{4 g \alpha \Delta T}{L}\right)^{1 / 2}=\text { the Brunt-Väisälä frequency. }
$$

In the two-dimensional case, when $y$ variations vanish, eqs (24) and the continuity equation can be written in component form as

$$
\begin{gathered}
\frac{\partial u}{\partial t}+\epsilon \mathbf{u} \cdot \nabla u-2 v=-\frac{\partial P}{\partial x}+\sigma^{1 / 2} \tilde{E} \nabla^{2} u \\
\frac{\partial v}{\partial t}+\epsilon \mathbf{u} \cdot \nabla v+2 u=\sigma^{1 / 2} \tilde{E} \nabla^{2} v \\
\frac{\partial w}{\partial t}+\epsilon \mathbf{u} \cdot \nabla w-2 F T=-\frac{\partial P}{\partial z}+\sigma^{1 / 2} \tilde{E} \nabla^{2} w \\
\frac{\partial T}{\partial t}+\epsilon \mathbf{u} \cdot \nabla T+2 F w=\sigma^{-1 / 2} \tilde{E} \nabla^{2} T \\
\frac{\partial u}{\partial x}+\frac{\partial w}{\partial z}=0 .
\end{gathered}
$$

Comparison of eqs (16) and (26) shows them to be identical when the following identifications are made:

$$
\begin{array}{lllllllll}
\text { System } \Omega_{S} & x & z & u & w & v & T & \tilde{E} & F \\
\text { System } S_{\Omega} & z & x & w & u & T & v & R & F^{-1} .
\end{array}
$$

In general the restriction must also be made that $\sigma=1$. However, for steady, linear flow, the analogy is valid for all Prandtl numbers since it can be incorporated into dependent variables by redefinition of these variables. The boundary conditions also must be appropriate for the analogy to be complete, and these boundary conditions can be made appropriate.

In reference 16 several examples of this analogy are presented. One example, waves in stratified or rotating fluids, which were discussed in the fully three-dimensional case in section 3 of the present paper, is shown to be mathematically identical in the two-dimensional case, when the identifications above are made. The analogy is much more profound than simply one between waves, however, since it applies in dissipative flows with boundaries. Furthermore, as noted by eqs (16) and (26), the analogy also applies in rotating flows when stratification modifies the flow and in stratified flows with modifying rotational effects. However, as discussed by Veronis [16], the analogy breaks down in fully three-dimensional cases.

\section{Nonlinear Considerations}

In this section a very brief account will be given of certain approaches to nonlinear problems in stratified and rotating fluids. This is an area of active interest because the equations of fluid dynamics are basically nonlinear and because relatively little is known about nonlinear phenomena compared with linear phenomena. However, it is also a very difficult area because the mathematical tools are limited.

Only one approach will be examined in any detail. The equations will be derived for finiteamplitude, two-dimensional steady flow of a stratified flow over an obstacle. These equations are derived and the method of solution briefly discussed because this approach is a unique one. Some of the other approaches to nonlinear problems use techniques common to other branches of fluid mechanics, and these will only be mentioned.

The nonlinear equations for steady, two-dimensional flow have been cast into a single equation for a stream function by Long [46] and into another equation by Yih [7]. These equations can be related simply under certain conditions, but each has been treated in a different fashion by its author. The equations will be derived and the method of solution of each briefly discussed. It should be noted that both authors have used inverse methods for solving problems. Boundary conditions are selected and a solution is obtained. Then the nonlinear problem which was solved is determined as the final step. Such a procedure is not without difficulty and controversy. There have been several papers in the literature discussing these difficulties [4, 45]. 


\subsection{The Equation of Long for Stratified Flow over an Obstacle}

For a two-dimensional, incompressible flow, the equations are independent of $y$. Equation (2.11) becomes two equations, incompressibility and continuity

$$
\begin{aligned}
& u \frac{\partial \rho}{\partial x}+w \frac{\partial \rho}{\partial z}=0 \\
& \frac{\partial u}{\partial x}+\frac{\partial w}{\partial z}=0
\end{aligned}
$$

while the equation of motion (2.12) becomes

$$
\begin{gathered}
\rho\left(u \frac{\partial u}{\partial x}+w \frac{\partial u}{\partial z}\right)=-\frac{\partial p}{\partial x} \\
\rho\left(u \frac{\partial w}{\partial x}+w \frac{\partial w}{\partial z}\right)=-\frac{\partial p}{\partial z}-g \rho .
\end{gathered}
$$

Here, the motion has been assumed steady as stated above, the equations are written in a nonrotating reference frame and the fluid is assumed inviscid so the $\mathbf{f}=0$.

Equations (3) and (4) can be rewritten as

$$
\begin{gathered}
\rho \frac{\partial}{\partial x}\left(\frac{u^{2}+w^{2}}{2}\right)+\frac{\partial p}{\partial x}+\rho w \zeta=0, \\
\rho \frac{\partial}{\partial z}\left(\frac{u^{2}+w^{2}}{2}\right)+\frac{\partial p}{\partial z}+\rho g-\rho u \zeta=0 .
\end{gathered}
$$

Here $\zeta$ is the $y$ component of the vorticity,

$$
\zeta=\frac{\partial u}{\partial z}-\frac{\partial w}{\partial x}
$$

From these two equations, the pressure is eliminated by cross differentiation; thus

$$
\frac{\partial \rho}{\partial z} \frac{\partial}{\partial x}\left(\frac{q^{2}}{2}\right)-\frac{\partial \rho}{\partial x} \frac{\partial}{\partial z}\left(\frac{q^{2}}{2}\right)-\frac{\partial \rho}{\partial x} g+w \frac{\partial}{\partial z}(\rho \xi)+u \frac{\partial}{\partial x}(\rho \xi)=0
$$

where $q^{2}=u^{2}+w^{2}$.

Equation (2) implies that a stream function can be defined as follows

$$
-u=\frac{\partial \psi}{\partial z}, w=\frac{\partial \psi}{\partial x}
$$

Then Eq. (2) is identically satisfied. Furthermore, when eqs (8) are used, eq (1) shows that the gradient of $\rho$ is parallel to the gradient of $\psi$, and therefore

$$
\rho=\rho(\psi) .
$$

It can be seen that for any functions of these qualities $f(\rho), g(\psi)$

$$
\left(u \frac{\partial}{\partial x}+w \frac{\partial}{\partial z}\right)\left\{\begin{array}{l}
f(\rho) \\
g(\psi)
\end{array}\right\}=\left\{\begin{array}{l}
\frac{d f}{d \rho} \\
\frac{d g}{d \psi}
\end{array}\right\}\left(u \frac{\partial}{\partial x}+w \frac{\partial}{\partial z}\right)\left\{\begin{array}{l}
\rho \\
\psi
\end{array}\right]=0 .
$$

In terms of the stream function, the vorticity becomes

$$
\zeta=-\nabla^{2} \psi
$$


With these observations, eq (7) can be written

$$
\left(u \frac{\partial}{\partial x}+w \frac{\partial}{\partial z}\right)\left[-\frac{d \rho}{d \psi} \frac{q^{2}}{2}+\rho \zeta-\frac{d \rho}{d \psi} g z\right]=0
$$

noting only that

$$
\begin{aligned}
\frac{\partial \rho}{\partial x} g=\frac{d \rho}{d \psi} \frac{\partial \psi}{\partial x} g=\frac{d \rho}{d \psi} w g & =\frac{d \rho}{d \psi}\left(u \frac{\partial}{\partial x}+w \frac{\partial}{\partial z}\right) g z \\
& =\left(u \frac{\partial}{\partial x}+w \frac{\partial}{\partial z}\right) \frac{d \rho}{d \psi} g z .
\end{aligned}
$$

Equation (12) can be integrated, since the derivative is along a streamline, to give

$$
-\frac{d \rho}{d \psi}\left(\frac{q^{2}}{2}+g z\right)+\rho \zeta=-H^{*}(\psi) \rho(\psi)
$$

where $H^{*}(\psi)$ is a function of $\psi$ to be determined from conditions upstream. Substitution for $q^{2}$ and $\zeta$ in terms of $\psi$ gives the single, nonlinear equation

$$
\frac{1}{\rho} \frac{d \rho}{d \psi}\left(\frac{(\nabla \psi)^{2}}{2}+g z\right)+\nabla^{2} \psi=H^{*}(\psi)
$$

This equation can be written in another form by noting that $H^{*}(\psi)$ has a specific form upstream, namely

$$
H^{*}(\psi)=\frac{1}{\rho(\psi)} \frac{d \rho}{d \psi}\left[\frac{U(\psi)^{2}}{2}+g z_{0}(\psi)\right]-\zeta_{0}(\psi)
$$

Thus

$$
\nabla^{2} \psi+\frac{1}{\rho} \frac{d \rho}{d \psi} \frac{(\nabla \psi)^{2}}{2}=-\zeta_{0}(\psi)+\frac{1}{\rho} \frac{d \rho}{d \psi}\left[\frac{U^{2}}{2}+g\left(z_{0}(\psi)-z\right)\right]
$$

A change of variables from $\psi$ to the upstream variable $z_{0}$, noting that

$$
\frac{d}{d \psi}=\frac{d z_{0}}{d \psi} \frac{d}{d z_{0}}=-\frac{1}{U} \frac{d}{d z_{0}}
$$

reduces eq (15) to the form

$$
\nabla^{2} z_{0}+\frac{1}{2} \frac{d}{d z_{0}}\left[\ln \rho U^{2}\right]\left[\left(\frac{\partial z_{0}}{\partial x}\right)^{2}+\left(\frac{\partial z_{0}}{\partial z}\right)^{2}-1\right]=\frac{g}{\rho U^{2}} \frac{d \rho}{d z_{0}}\left(z_{0}-z\right) .
$$

The transformation (16) can be used only when the upstream velocity $U$ does not change sign. In this form, Long [46] observes that the equation becomes linear if $\rho U^{2}=$ constant and

$$
\frac{d \rho}{d z_{0}}=\text { constant }
$$

Then, with $\delta=\left(z_{0}-z\right)$, eq (17) becomes

where

$$
\nabla^{2} \delta+\sigma^{2} \delta=0
$$

$$
\sigma^{2}=\frac{g}{\rho U^{2}}\left|\frac{d \rho}{d z_{0}}\right| .
$$

Long [46] solved for the flow over obstacles of infinitesimal height first. The resulting solution was found to satisfy eq (18) also for obstacles of finite height. However, the streamline along the plane $z=0$ upstream, which is found to describe an obstacle of finite height, specifies 
the shape of the obstacle. Subsequent work has extended the analysis to obtain solutions for flows over thin obstacles of specified shape and arbitrary height [45].

This general method of approach has been extended by Long [45] to other two-dimensional, steady, finite-amplitude systems. For example, he has derived a single nonlinear equation for the stream function for axisymmetric motion with a body forre and for the stream function in two-dimensional, steady motion in a rotating fluid. In the latter case, a linear form of the nonlinear equation can be obtained as described above, and this equation is exactly eq (18) with a different expression for $\sigma^{2}$, but with $\delta$ also being the deflection of the stream line from its upstream height.

\subsection{The Equation of Yih for Stratified Flow over an Obstacle}

Another single nonlinear equation has been derived by Yih [7] from eqs (1)-(4), and he has systematically examined the implications of this equation. In this subsection the equation of Yih will be derived.

Again, eqs (1)-(4) are the starting point for the derivation. Yih [7] observes that the inertia effect of a variable density (mentioned in section 2 of this report) can be accounted for, defining new velocity variables $u^{\prime}, w^{\prime}$, as follows:

$$
\begin{aligned}
& u^{\prime}=\sqrt{\frac{\rho}{\rho_{0}}} u \\
& w^{\prime}=\sqrt{\frac{\rho}{\rho_{0}}} w
\end{aligned}
$$

where $\rho_{0}$ is a constant reference density. Then Eqs (1)-(4) become

$$
\begin{gathered}
u^{\prime} \frac{\partial \rho}{\partial x}+w^{\prime} \frac{\partial \rho}{\partial z}=0 \\
\frac{\partial u^{\prime}}{\partial x}+\frac{\partial w^{\prime}}{\partial z}=0 \\
\rho_{0}\left(u^{\prime} \frac{\partial u^{\prime}}{\partial x}+w^{\prime} \frac{\partial u^{\prime}}{\partial z}\right)=-\frac{\partial p}{\partial x} \\
\rho_{0}\left(u^{\prime} \frac{\partial w^{\prime}}{\partial x}+w^{\prime} \frac{\partial w^{\prime}}{\partial z}\right)=-\frac{\partial p}{\partial z}-g \rho .
\end{gathered}
$$

(Note that these equations follow immediately from the observation made in eq (10) with

$$
\left.f(\rho)=\sqrt{\rho / \rho_{0}} .\right)
$$

Equations (22) and (23) can be rewritten as

$$
\begin{gathered}
\rho_{0}\left(u^{\prime} \frac{\partial}{\partial x}+w^{\prime} \frac{\partial}{\partial z}\right) \frac{u^{\prime 2}}{2}=-u^{\prime} \frac{\partial p}{\partial x} \\
\rho_{0}\left(u^{\prime} \frac{\partial}{\partial x}+w^{\prime} \frac{\partial}{\partial z}\right) \frac{w^{\prime 2}}{2}=-w^{\prime} \frac{\partial p}{\partial z}-g \rho w^{\prime}
\end{gathered}
$$

when eq (22) is multiplied by $u^{\prime}$ and (23) by $w^{\prime}$. Addition of eqs (24) and (25), with the observation that

$$
g \rho w^{\prime}=g \rho\left(u^{\prime} \frac{\partial}{\partial x}+w^{\prime} \frac{\partial}{\partial z}\right) z=\left(u^{\prime} \frac{\partial}{\partial x}+w^{\prime} \frac{\partial}{\partial z}\right) g \rho z
$$

yields the relation

$$
\left(u^{\prime} \frac{\partial}{\partial x}+w^{\prime} \frac{\partial}{\partial z}\right)\left[\rho_{0} \frac{u^{\prime 2}+w^{\prime 2}}{2}+p+g \rho z\right]=0 .
$$


This relation implies that the quantity

$$
H=\rho_{0} \frac{u^{\prime 2}+w^{\prime 2}}{2}+p+g \rho z,
$$

known alternately as the Bernoulli quantity [7] and the "total head" [4], is a constant along a stream line. It will, however, vary from stream line to stream line. Yih [7] defines a stream function $\psi^{\prime}$ based upon the velocities $u^{\prime}$ and $w^{\prime}$ as follows:

$$
u^{\prime}=\frac{\partial \psi^{\prime}}{\partial z}, w^{\prime}=-\frac{\partial \psi^{\prime}}{\partial x}
$$

Then eq (21) is identically satisfied, and since $H$ is a function depending upon the stream line, $H=H\left(\psi^{\prime}\right)$.

With the definitions and observations made above, the single nonlinear equation of Yih can be derived. Equations (22) and (23) can be written as:

$$
\begin{gathered}
\rho_{0} \frac{\partial}{\partial x}\left(\frac{u^{\prime 2}+w^{\prime 2}}{2}\right)+\frac{\partial p}{\partial x}+\rho_{0} w^{\prime} \zeta^{\prime}=0 \\
\rho_{0} \frac{\partial}{\partial z}\left(\frac{u^{\prime 2}+w^{\prime 2}}{2}\right)+\frac{\partial p}{\partial z}+\rho g-\rho_{0} u^{\prime} \zeta^{\prime}=0
\end{gathered}
$$

where

$$
\zeta^{\prime}=\frac{\partial u^{\prime}}{\partial z}-\frac{\partial w^{\prime}}{\partial x}
$$

is the vorticity based upon the velocities $u^{\prime}, w^{\prime}$. Multiplying eq (28) by $d x$ and eq (29) by $d z$ and adding yields

$$
d\left[\rho_{0} \frac{u^{\prime 2}+w^{\prime 2}}{2}+p+\rho g z\right]-\rho_{0} \zeta^{\prime} d \psi^{\prime}-z g d \rho=0
$$

where

$$
d=d x \frac{\partial}{\partial x}+d z \frac{\partial}{\partial z}
$$

Since

$$
H=H\left(\psi^{\prime}\right) \text { and } \zeta^{\prime}=\nabla^{2} \psi^{\prime}
$$

eq (30) becomes

$$
\nabla^{2} \psi^{\prime}+\frac{z g}{\rho_{0}} \frac{d \rho}{d \psi^{\prime}}-\frac{1}{\rho_{0}} \frac{d H}{d \psi^{\prime}}=0
$$

and this is the equation of Yih.

Yih systematically examined solutions to this equation when

$$
\frac{d \rho}{d \psi^{\prime}} \text { and } \frac{d \mathrm{H}}{d \psi^{\prime}}
$$

are linear functions of $\psi^{\prime}$; then eq (31) is linear and classes of solutions to the original nonlinear equation are found. For details of these solutions, see references [7].

\subsection{Other Nonlinear Problems}

The purpose of this subsection will be to mention some other nonlinear problems in stratified or rotating fluids. In some cases the mathematical development is exactly the same as in 
other areas of fluid dynamics; in others the mathematical techniques have been developed for problems in different areas and have subsequently been applied to flows of stratified or rotating fluids.

The free surface flow of a shallow layer of homogeneous fluid over an obstacle is an example of a stratified (layered) flow in which the governing equations are exactly the same as the onedimensional, compressible-flow equations for a polytropic gas in which the gas pressure is proportional to the density squared. This analogy has been known for some time [47], and the governing equations are hyperbolic [26] as defined in section 3 of this report. Perhaps the most interesting aspect of this analogy is that an identification can be made between the internal Froude number in the stratified flow and the Mach number in the compressible flow [7, 45, 47].

The internal Froude number is defined in eq (2.5); here it is rewritten slightly as

$$
F=\frac{U}{\left(\frac{\rho_{1}-\rho_{2}}{\rho_{1}} g L\right)^{1 / 2}}
$$

where $U$ is the uniform upstream velocity in the shallow layer of fluid, $\rho_{1}$ is the density and $L$ the thickness of the layer of fluid and $\rho_{2}$ is the density of the fluid overlaying this layer. The velocity

$$
\left(\frac{\rho_{1}-\rho_{2}}{\rho_{1}} g L\right)^{1 / 2}
$$

is that of infinitesimal long waves on the layer of fluid. The Mach number is defined in eq (2.10) as

$$
M=U / c_{0}
$$

where $U$ is the characteristic flow velocity and $c_{0}$ is the speed of sound waves.

When the Froude number is less than one, the flow is said to be subcritical; when $F>1$, supercritical and when $F=1$, critical. In each of these cases, the surface elevation of the flow over the obstacle changes in character. In subcritical flows, the surface elevation decreases from its upstream value as the fluid flows over the obstacle. In the supercritical case, the surface elevation increases as the flow passes over the obstacle. When the flow becomes critical, phenomena known as hydraulic jumps or bores occur. For a description of the flow regimes as a function of internal Froude number, see references [45] and [4].

The point to note here is the analogy between stratified flow and compressible flow. The Froude number is the parameter which delineates various regimes exactly as the Mach number delineates regimes of flow in gasdynamics. The phenomenon of the hydraulic jump is exactly analogous to the shock wave in gasdynamics. Furthermore, for a variety of flow conditions, the mathematical formulation and solutions are identical.

An area of rather intense study presently is that of nonlinear waves [26, 48, 49]; nonlinear wave phenomena appear in many fields, and much of the mathematical apparatus is applicable to all of these fields. Formation of shock waves in compressible flows or hydraulic jumps in water-wave theory is an example of a nonlinear wave arising from a hyperbolic equation. More recent attention has been focussed on nonlinear, dispersive waves, where the nonlinear tendency to steepen is balanced by the dispersive tendency to spread them. Examination has been made of both periodic (cnoidal) waves and nonperiodic (solitary) waves in such systems.

Another particularly active problem area in the theory of nonlinear, dispersive waves is that of wave-wave interactions. Conditions are examined for which the nonlinear terms in the equations of motion can permit resonant coupling between wave modes of the linear system. One example where such coupling can occur is in the interaction between internal and surface waves [49]. Another example, which has been intensively investigated recently, is that of coupling between laser radiation and oscillation modes in a plasma; these so-called parametric instabilities have been examined for possible application in laser-driven fusion [50].

As final examples of nonlinear problems, boundary layers in rotating fluids [9] and nonlinear convection in stratified fluids [4] are mentioned. In the next section of this report some of the linear features of these problems will be discussed.

\section{Transport and Diffusion Processes}

In this section some interesting problems will be discussed related to transport and diffusion processes in a stratified or rotating fluid. Since the work in this area is immense, only three problems have been selected for discussion, and even these are discussed only briefly. However, 
it is felt that this presentation will convey the type of studies being carried out. Reference should be made to the monographs and papers cited in this section.

The three problems to be discussed are contained, rotating fluids, convection in stratified fluids, and turbulence in stratified or rotating fluids. The problems of contained, rotating fluids has been reviewed extensively by Greenspan [9], who has also been a major contributor to the research in this area. A more recent review of the problem of "spin-up," which is a result of viscous effects and boundaries, has been given by Benton and Clark [14]. The analogy between rotating and stratified fluids [16], discussed in section 4, allows some of the results from rotating fluids to be carried over to stratified fluids. Convection in stratified fluids covers a large number of phenomena. Turner [4], who has been a major contributor in this area, has recently reviewed these phenomena. Finally, turbulence, which in many respects acts as a diffusive effect, in stratified fluids will be discussed. Turner [4] has recently described some important features of turbulence in stratified fluids. Turbulence in the atmosphere has been discussed by Lumley and Panofsky [55], and Phillips [5] has reviewed turbulence in the ocean. Work in this very important area has been growing rapidly.

\subsection{Contained Rotating Fluids}

The most comprehensive review of flow phenomena in rotating fluids is the monograph of Greenspan [9]. In that book he presents a systematic development of the formulation and solution of problems concerned with contained rotating fluids. Boundaries and viscous boundary layers produce such a dominant effect in these fluids, they are discussed briefly here. First the basic linear equations are derived. Then the Ekman layer, a fundamental boundary layer in rotating fluids, is discussed. Finally, the phenomenon known as spin-up is mentioned.

The basic equations for the analysis of contained rotating flows are eqs (2.11) and (2.12). The fluid is assumed to be homogeneous, incompressible and viscous; therefore, eq (2.11) becomes $\rho=\rho_{0}$ and

Equation (2.12) becomes

$$
\nabla \cdot \mathbf{u}=0 .
$$

$$
\rho_{0} \frac{D \mathbf{u}}{D t}+2 \rho_{0} \boldsymbol{\Omega} \times \mathbf{u}+\nabla P=\rho_{0} \nu \nabla^{2} \mathbf{u} .
$$

Here $P$ is the reduced pressure defined earlier, the body force due to gravity has been dropped because the fluid is homogeneous and the force $\mathbf{f}$ in eq (2.12) has been taken to be due to viscosity.

The characteristic length, time and velocity scales, $L, \Omega^{-1}$ and $U$ are chosen to nondimensionalize the variables. Therefore $\mathbf{r}, t, \mathbf{u}, \boldsymbol{\Omega}=\Omega \mathbf{i}_{z}$ and $P$ are all scaled by these quantities to become, $L \mathbf{r}, \Omega^{-1} t, U \mathbf{u}, \Omega \mathbf{i}_{z}$ and $\rho_{0} \Omega U L P$, and eqs (1) and (2) become in dimensionless form

$$
\nabla \cdot \mathbf{u}=0
$$

$$
\frac{\partial \mathbf{u}}{\partial t}+\epsilon \mathbf{u} \cdot \nabla \mathbf{u}+2 \mathbf{i}_{z} \times \mathbf{u}+\nabla P=E \nabla^{2} \mathbf{u}
$$

where

$$
\epsilon=\frac{U}{\Omega L}
$$

is the Rossby number and

$$
E=\frac{\nu}{\Omega L^{2}}
$$

is the Ekman number. When the equations are linearized, $\epsilon=0$, and eq (4) becomes

$$
\frac{\partial \mathbf{u}}{\partial t}+2 \mathbf{i}_{z} \times \mathbf{u}+\nabla P=E \nabla^{2} \mathbf{u}
$$

Equations (3) and (5) can be used to derive a single equation for the pressure. When the curl of eq (5) is taken, the equation for the vorticity $\omega=\nabla \times \mathbf{u}$ is obtained

$$
\left(\frac{\partial}{\partial t}-E \nabla^{2}\right) \nabla \times \mathbf{u}=2 \frac{\partial}{\partial z} \mathbf{u} .
$$


When the divergence of eq (5) is taken, again using eq (3),

The operator

$$
\nabla^{2} P=2 \mathbf{i}_{z} \cdot(\nabla \times \mathbf{u}) .
$$

$$
\left(\frac{\partial}{\partial t}-E \nabla^{2}\right)
$$

is applied to eq (6) and eq (5) is used to obtain

$$
\left(\frac{\partial}{\partial t}-E \nabla^{2}\right)^{2}(\nabla \times \mathbf{u})=2 \frac{\partial}{\partial z}\left[-\nabla P-2 \mathbf{i}_{z} \times \mathbf{u}\right]
$$

Then the $z$ component of this equation, using eq (7), gives the desired equation for the pressure.

$$
\left(\frac{\partial}{\partial t}-E \nabla^{2}\right)^{2} \nabla^{2} P=-4 \frac{\partial^{2} P}{\partial z^{2}} .
$$

Some idea of the difficulty of problems of this type is obtained by examination of eq (9).

A very fundamental role in the theory of rotating flows is played by the Ekman layer. In this layer a balance is struck between viscous and Coriolis forces. In the simplest case, this balance can be seen by the following model. Consider a half-space of fluid $z \geq 0$, rotating with constant angular velocity $\Omega$. Assume that a uniform velocity $u=U, v=0$ is imposed at the surface $z=0$, and look for a steady-state solution which is independent of the horizontal coordinates $x$ and $y$. The scaled eqs (5) become

$$
-2 v=E \frac{d^{2} u}{d z^{2}}, 2 u=E \frac{d^{2} v}{d z^{2}} .
$$

The solution to eqs (10) which satisfies the boundary conditions is

$$
\begin{aligned}
& u=\exp \left(-\frac{z}{\sqrt{E}}\right) \cos \frac{z}{\sqrt{E}} \\
& v=\exp \left(-\frac{z}{\sqrt{E}}\right) \sin \frac{z}{\sqrt{E}} .
\end{aligned}
$$

From the continuity equation (3) and the third of eqs (5) it is found that the vertical velocity $w=0$ and that the pressure is constant.

Two important features of the Ekman layer can be seen from the solution (11). First, the Ekman layer is a boundary layer of thickness $\sqrt{\frac{\nu}{\Omega}}$; the velocity, which is unity (in nondimensional units) at $z=0$, decays exponentially over this thickness. Second, the velocity vector rotates as $z$ increases. Plotted in polar coordinates, the velocity forms a spiral shape, the socalled Ekman spiral $[9,16]$.

Examination of the dynamics of the Ekman layer under more realistic conditions shows that the vertical velocity is not zero as determined by the simple model above [9]. Rather, the vertical velocity increases exponentially through the Ekman layer from zero at $z=0$ to a value of $-\mathrm{E}^{1 / 2}$. When $E$ is small, as is usually the case, this vertical velocity is small. However, this suction velocity dramatically affects the inviscid flow.

Spin-up is one problem in which the Ekman layer plays a fundamental role. Consider a container filled with a homogeneous fluid and rotating with the fluid about a vertical axis at a constant angular velocity. Then the angular velocity of the container is suddenly increased to a new value and maintained at that value. Along the top and bottom surfaces of the container Ekman layers form. In each layer the centrifugal force is too great for the prevailing pressure gradient, and the fluid is driven radially outward. By continuity, the suction velocity into each layer arises, and this velocity produces secondary circulations within the contained fluid, including additional boundary layers of a different nature along the vertical walls. Ultimately (after a time scale $L / \nu^{1 / 2} \Omega^{1 / 2}$, determined by the length scale $L$ and the Ekman suction velocity $E^{1 / 2} \Omega L$ ) these secondary flows lead to a state of solid body rotation of the fluid at the new angular velocity. This rather complex process is called spin-up [9, 14]. 


\subsection{Convection in Stratified Fluids}

In a recent book, Turner [4] has given a review of many interesting and practical convective phenomena in stratified fluids. In this subsection, following Turner, a very brief discussion of these phenomena will be presented. It will address convection from isolated sources of buoyancy, convection from heated surfaces and so-called double diffusive phenomena.

\section{a. Buoyant Thermals and Plumes}

In many situations the buoyancy in a fluid can supply the energy for the mean motion within the system. An example of such a situation is the thermal, well known to meteorologists and pilots, in which a mass of warmer, lighter air rises due to buoyancy in cooler, more dense surroundings. Another example is the thermal plume created when hot water from a power plant is discharged into a waterbody, the discharge water rising in the cooler ambient water. (Effluents from chimneys in the atmosphere also often fall into this category when the effluent is more buoyant than the surrounding air.)

These two examples display the properties of two broad categories into which flows of this nature have been divided by 'Turner [4]. The first, called the thermal, is characterized by a sudden release of buoyancy within a fluid. The buoyancy remains confined to a limited amount of fluid, which, as it rises, loses connection with the source that produced it. The second, called a plume or buoyant jet, is characterized by a continuous source of buoyancy. Rising buoyant fluid is always connected by additional buoyant fluid to its source.

The driving force in both of these types of motion is the buoyant force. Lighter fluid will be buoyed upward in its surrounding heavier fluid. (Likewise, heavier fluid will fall in lighter surroundings.) This fluid will rise causing a mean convective motion and smaller scale motions called entrainments near the interface between the buoyant fluid and its surroundings. Generally, the entrainment is a turbulent process in which surrounding fluid is captured into the thermal or plume fluid. Entrainment causes the volume of buoyant fluid to be increased, but the net buoyancy of each fluid element to decrease relative to the surroundings.

The mathematical techniques, in addition to numerical ones, generally used to analyze buoyant thermals and plumes are similarity methods. A major consideration in the analysis of these flows is the entrainment and mixing of the ambient fluid, since entrainment determines both the mean size and the net upward flow velocity of the thermal or plume. Other complicating features in the analysis are nonhomogeneous ambient media and an ambient steady or unsteady mean cross flow. The steady cross flow causes a plume to be "bent over" in the direction of flow, changing the mean properties of the plume and often the character of the entrainment as well.

\section{b. Convection from Heated Surfaces}

A fundamental problem with a long history in the theory of convective flows is the Bénard problem [52]. Fluid, contained between two horizontal surfaces, is heated from below. The increased buoyancy tends to destabilize the fluid, and when the dissipative effects of thermal conduction and viscosity are overcome, the fluid will overturn. The parameter which governs this stability problem is the Rayleigh number $[4,52]$

$$
\mathrm{Ra}=\frac{g \alpha \Delta T d^{3}}{\mathrm{~K} \nu} .
$$

Here, all of the symbols have their usual meanings. $\Delta T$ is the temperature difference across the fluid layer of thickness $d$ and $\mathrm{K}$ is the thermometric conductivity. The results of the experiments and analysis of this problem show two main features. First, a critical Rayleigh number must be exceeded before instability occurs; this implies that a certain temperature difference must be exceeded for a given fluid and layer thickness. Second, when convection sets in, the motion is organized in regular, steady cellular patterns. Chandrasekhar [52] has given an encyclopedic review of the linear aspects of this instability problem, including the effects of rotation and magnetic fields. Subsequent work has concentrated on finite-amplitude aspects of the convection [4].

Turner [4] also reviews other studies of convection caused by heated surfaces. These include intermittent thermal or plume formation at a horizontal surface, so-called penetrative convection (where convection initiated in an unstable region penetrates into an adjacent stable region), and convection layers induced at vertical or sloping boundaires. 
An area of study in convective phenomena, which is of relatively recent origin, is doublediffusive phenomena [4]. A fluid is considered for which the density of the fluid is determined by the concentration of two properties, which have differing diffusivities. In the ocean, for example, the temperature and the salinity are the two properties; each affects the density of the water by its concentration, and the salt and temperature have different diffusivities. These phenomena appear to be applicable to more problems than only oceanic ones.

In the ocean, the concentration of salt and the temperature of the water determine its density. An increased salinity increases the density whereas an increased temperature decreases it. Therefore, the possibility of interesting double-diffusive instabilities arises. For example, if colder, less saline water underlies warmer, more saline water, such that the density of the lower layer is greater, then the fluid should be stable. However, if a fluid parcel from the lower layer is displaced upward, due to the difference in diffusivities, the parcel heats up while retaining its lower salinity. Then the parcel is less dense than the surrounding fluid and rises, leading to an unstable situation. This instability is the mechanism for a "salt fountain," which is the flow through a heat-conducting pipe of the colder, less-saline fluid to the top of the overlying layer of warmer, saline water (or the opposite flow).

When cold, fresh water is above hot, saline water, such that the density increases downward, another type of instability can occur. A displaced fluid element of hot saline fluid will be more dense than its surroundings and will tend to sink. In the absence of any diffusion processes, the fluid element would oscillate at the local Brunt-Väisälä frequency. However, because of the relatively large thermal diffusion, the displaced element would cool somewhat before reaching its original equilibrium position, and, therefore, would plunge lower than without diffusion. Heating during the lower portion of the oscillation cycle would then cause the fluid parcel to rise higher during its upward motion. Repeated cooling and heating during the oscillation cycle causes oscillations of growing amplitude, a process known as overstability.

These two instabilities can lead to interesting phenomena known as salt fingers and microstructure. Salt fingers are long vertical columns of warm salt water in cooler, less saline water; they are believed to result from the former instability. Microstructure is a series of horizontal layers of fluid of relatively uniform properties separated by rather abrupt changes in these properties. It is believed that some microstructure is a result of double diffusion convection [4].

\subsection{Turbulence}

Turbulence is one of the most prevalent phenomena within fluids. Its motions are often mainly responsible for the transport and diffusion of mass, momentum and energy within these fluids. Because of this prominent position, it has been examined extensively. But, because of its inherently nonlinear and random nature, progress in understanding and modeling it has been slow. In this subsection no attempt will be made to review the extensive literature in this area; rather, a very brief discussion will be given to indicate its importance in the study of stratified and rotating fluids.

Turbulence in stratified fluids is an especially interesting and challenging problem. Mixing of a stably stratified fluid requires the expenditure of energy, and, therefore, vertical turbulent mixing is inhibited by stratification. The parameter which governs this interaction is the local Richardson number. The local Richardson number compares the suppression of turbulence by the mean buoyancy forces to the production of turbulence by Reynolds stresses [5].

Turbulence in the atmosphere, which is generally stratified, has been and continues to be an area of active interest [53-55]. The atmospheric boundary layer is also influenced by rotational effects [56]. In fact, a model of this boundary layer is an Ekman layer in which Coriolis forces are balanced by Reynolds stresses (rather than viscous stresses as described earlier).

Similarly, turbulence in the ocean and in lakes is a dominant influence on their structures [5]. The interaction between turbulence and buoyancy gradients is often manifested by a rather sharp interface between a turbulently mixed region and an adjacent stably stratified region $[4,5]$. It is this interaction which has been used to explain the distinctive temperature structure in temperate lakes, for example [57].

For many purposes in geophysical applications, including the two specific ones cited above, turbulence acts as a diffusive process. Then the effects of turbulence are modeled by mixing-length theories, where the nonlinear, random mixing is related to the mean-flow gradients through turbulent diffusion coefficients. For discussion of the modeling and the effects of turbulence, the references cited in this section should be consulted. 


\section{Some Applications}

The basic concepts in studies of stratified and rotating fluids have wide applicability, particularly in the area of geophysics. Some of the applications have already been mentioned in the introduction and at other parts of this report. In this section, a brief summary of the geophysical applications is presented. This summary will not be complete, but will convey the rich variety of interesting problems and results.

\subsection{Physical Oceanography}

In the ocean a very large variety of fluid motions occur, ranging in length scale from millimeters to tens of thousands of kilometers. It is the largest scale circulations which differ most from classical fluid flows because curvature and rotation of the earth, stratification produced by solar heating and even the differential gravitational effects producing tides dominate these motions [58]. However, even the smaller scale motions are profoundly influenced by rotation and stratification.

The ocean is a very thin layer on a nearly spherical globe. In the deepest region, the Marianas Trench, the ocean is about $12 \mathrm{~km}$ deep. The radius of the earth is about $6400 \mathrm{~km}$, so that the ocean depth is less than 0.2 percent of the radius of the earth [59]. For consideration of many fluid motions the curvature of the earth can be neglected; but for larger curculations, the curvature must be considered. A common approximation used to simplify analysis of oceanographic circulations is the " $\beta$-plane approximation." The fact is used that the depth $\Delta$ of the ocean is much less than the radius $R$ of the earth. It is assumed that the lateral length scale $L$ for motions is much greater than the depth and that $L<<R$, the most questionable part of the approximation [58]. Then the equations of motion for the sheet of fluid on the rotating globe are reduced to equations for a thin sheet of fluid in a rotating tangent plane.

The waters at the equator are heated by solar radiation and those at the poles undergo net radiational cooling [59]. In the absence of rotation, therefore, the more buoyant surface fluid near the equator would spread toward the poles. However, the Coriolis force curves this spreading, producing large clockwise eddies in the northern hemisphere and counterclockwise eddies in the southern hemisphere. These large circulations are also known as gyres.

Both heating and salinity determine the density and therefore the buoyancy of the fluid. The temperature and salinity combine generally to form a very distinctive density profile in which an approximately uniform-density layer overlays another layer of slowly varying properties. The narrow region which separates these layers, where the temperature varies rapidly and the Brunt-Väisälä frequency reaches a maximum with depth, is called the thermocline. This stratification has important consequences for oceanic circulations.

Another very important influence on the motions of all scales in the ocean are the winds $[60,9]$. The large scale, nearly steady atmospheric circulations are discussed in the subsection on meteorology. They are driven by solar heating and the earth's rotation in much the same way as the oceans. These winds transfer mechanical energy to the ocean surface by generating currents and waves and receive heat, primarily through evaporation from the ocean. The large-scale oceanic circulations are driven in a major way by this interaction [60].

The land masses also have a major influence on the oceanic gyres. The continents act as boundaries inhibiting the free circulations. The boundary layers at these surfaces influence the overall fluid motions in important ways [61]. On smaller scales, the shape of land masses cause many interesting phenomena such as wave diffraction and wave breaking.

The gravitational influence of the sun and moon also affect the largest scale motions in the ocean, forming the well-known oceanic tides. Tidal theory considers long wavelength waves in shallow water on a rotating globe and has been reviewed recently by Hendershott and Munk [62].

In addition to these large scale motions, many smaller scale motions occur. Both internal and inertial waves occur within the ocean $[5,6]$ and surface waves are created by the wind $[5,47]$. Also, at the surface, evaporation and condensation are important interchanges with the atmosphere, altering the local salinity and providing a major source for energy transfer with the atmosphere [60].

Finally, the pervasive influence of turbulence is felt. In the upper ocean above the thermocline, it is a major cause of mixing and diffusion [5, 60]. Likewise, near all land masses, turbulent boundary layers are dominant [61]. Most of the upwelling motions, the rise of nutrientrich lower waters, are influenced by turbulence. Even the deep oceanic currents are subject to its nonlinear, random processes. 
Many of the dynamical considerations associated with oceanography are also associated with limnology. The differences arise primarily in the large scale motions, where lake circulations are determined by the confines of the basin. Lakes are also generally composed of fresh water, but even this is not always the case.

Compared with the differences, the number of dynamical similarities is great [19]. Rotation affects the dynamical behavior of larger lakes $[19,13]$. As in the ocean and the atmosphere the horizontal dimensions of motions are much larger than the vertical dimension. Lakes are bounded by the atmosphere and derive mechanical energy for driving circulations from wind frictional effects. Thermal energy is provided by the sun, and thermal interchange takes place with the atmosphere. Finally, as in the ocean and the atmosphere, turbulence is a dominant influence on the motions.

In the remainder of this subsection some of the interesting features of modeling lake motions will be discussed. As with the other subsections on applications, no attempt can be made to be complete.

The Reynolds number determines when a flow is laminar and when it makes a transition to turbulence: for a Reynolds number greater than a critical value, the flow is turbulent. For a length scale equal to the depth, the velocity must exceed a minimum value for turbulence. For a very shallow lake of $100 \mathrm{~cm}$, the flow velocity must exceed $0.03 \mathrm{~cm} / \mathrm{s}$ [19]. Clearly then, the turbulent state is the natural one in a lake.

Because turbulence is a random process, statistical averages in time or space are taken to describe the character of the turbulence. This averaging brings up a very fundamental question of separating turbulent motions from longer-time-scale (or length-scale) motions associated with mean flows [55]. Assuming that this separation can be made, the nonlinear nature of the turbulence brings up a second fundamental problem, the so-called closure problem. If statistical averages of the equations of motion are taken, the nonlinear terms always couple lower order statistical correlations to higher order ones.

In applications such as limnology (and oceanography and meteorology), it is generally assumed that second order correlations are related to gradients of the mean flow through coefficients of eddy diffusivity [19]. When this is done, the effects of turbulence arise in the equations of motion as additional diffusion terms. A major problem, then, is to determine both the size and the variation of these coefficients with position and properties of the flow [19, 57].

The primary source of mechanical energy for currents in lakes is the wind, introducing both mean circulations and mixing into the main body of the water. The wind-driven circulations begin with water movement in the downwind direction, but since the lake is enclosed, a return current is also induced. Both influents and effluents can also affect circulation patterns, however. These circulation patterns are also profoundly affected by rotational and stratification effects.

In larger lakes the equations of motion and many of the approximations used in analyzing these equations are the same as those used in oceanography. In particular, geostrophy and Ekman layers are very important in interpreting limnological flows [19, 13].

Also, stratification is a dominant influence in the hydrodynamics of lakes. The stratification in lakes arises primarily from surface heating, and, therefore, lakes undergo an annual cycle in overall stratification $[19,57]$. During the spring and summer when the solar and atmospheric heat input is largest the degree of stratification, or the stability of the lake increases, and during fall and winter the lake cools and destabilizes.

Stratification affects both the current in the lake and the waves which the lake can sustain. In the absence of stratification currents in a lake would vary smoothly from a maximum value near the surface to zero at the bottom. In the presence of stratification the flow velocity will generally reverse in direction as a function of depth. Such behavior is very important in analyzing the mechanics of lakes.

As in the ocean a thermocline is often formed within a lake. Because of the seasonal variation in the stratification, the depth and thickness of the thermocline vary considerably. Because of the thermocline, however, a lake is often modelled as two superposed layers of fluid of different density, the so-called two-layer model. Internal waves, as well as surface waves, can be sustained within a lake due to the stratification.

Because a lake is confined by its basin, it has normal modes of oscillation. The term seiche is used to describe stationary oscillations of a lake or a large independent portion of a lake. Seiches have been studied extensively. Both stratification and rotation have important effects on seiches $[19,13]$. 


\subsection{Meteorology}

The atmosphere also is a very thin layer of fluid on the almost spherical earth, the thickness of the significant portion of it being about $10 \mathrm{~km}$, or a fraction of 1 percent of the radius of the earth. Therefore, horizontal dimensions of motions are generally much larger than the vertical dimension. The stratification of the atmosphere enhances this behavior since it tends to inhibit vertical motions.

In an interesting review of meteorology, Sutton [63] concentrates on three systems of motion which are determined by "their scales of horizontal length and their mean 'lives'." The largest motions are "huge quasi-permanent wind belts of the general circulation." The intermediate ones are the "smaller, short-lived cellular-like systems (highs and lows) that determine the day-by-day occurrence of weather." The smallest and shortest-lived motions are "the transient eddies that are responsible for turbulent transfer processes in the earth's boundary layer."

The general circulation is determined by the same large-scale effects that determine oceanic circulations, namely rotation, curvature of the earth, heating at the equator and cooling at the poles. These large scale circulations are associated with climate, the monthly or seasonal properties of the atmosphere averaged over long periods of time. Stewart [60] describes the atmosphere as a heat engine, receiving solar heat from the surface of the earth and the ocean, converting a portion of it to mechanical energy and discharging the remaining heat at lower temperatures. As mentioned in subsection 7.1 the wind of the atmosphere drives the oceanic circulations. In turn the ocean supplies a major fraction of the heat which the atmosphere uses in producing mechanical energy, some through direct heat transfer to the atmosphere, but most through evaporation at the surface of the ocean. The evaporation-condensation cycle is a major source of energy transfer between the ocean and atmosphere, which are very intimately linked dynamically [60].

Models for weather prediction have been reviewed by Sutton [63] and by Phillips [64] and include the effects of the earth's rotation, curvature of the earth and stratification of the atmosphere. Often the vertical equation of motion is replaced by the equation for hydrostatics, the so-called hydrostatic system. A further approximation sometimes made is that the horizontal equations can be replaced by a balance between pressure and the Coriolis forces, the geostrophic relation. An energy equation to account for the important energy fluxes in the atmosphere is included, and account must be taken of the water-vapor content of the air. The full equations or the various approximate sets are then integrated numerically, often over a 24-hour period. Extended forecasts have shown that a small predictive capability exists beyond 10 days [64].

Momentum and energy transfer between the earth and the atmosphere are important in about the lower 1000 meters of the atmosphere, the atmospheric boundary layer [53-56]. In this region the turbulent diffusion is balanced by the Coriolis force so that this layer is an Ekman layer. Radiative energy absorption takes place primarily at the surface of the earth so that atmospheric heating occurs from the bottom. (This is to be contrasted with the ocean where heating takes place at the top.) Therefore, thermal energy transfer and buoyancy effects are dominant in the atmospheric boundary layer.

The study of the turbulent boundary layer is called micrometeorology. Important features of this study are the small scale, turbulent mass, momentum and energy transfer. Micrometeorology has become a prominent area of study as concern has increased about diffusion of pollutants in the lower atmosphere.

\subsection{Thermal Discharges}

A major problem of practical concern in recent years is that of discharges into the atmosphere or into bodies of water. In subsection $6.2 \mathrm{a}$ on Buoyant Thermals and Plumes, some fundamental fluid-mechanical features of buoyant discharges (usually produced by heating the effluent and therefore called thermal discharges) and techniques for calculating these features were reviewed. Here, some general comments on the behavior of thermal discharges and the practical considerations related to these discharges will be made.

The mixing between a thermal discharge or plume and the ambient fluid can be viewed to take place in two parts. Near the outfall, the entrainment and mixing is dominated by the turbulence induced by the discharge, and this region is called the near field. In the far field, the entrainment and mixing are dominated by the naturally occurring turbulence in the ambient fluid.

The near field has been discussed in a variety of papers [4, 65-68]. If the effluent is discharged with a substantial initial momentum, the effects of buoyancy can often be neglected in the immediate vicinity of the outfall; then standard results on the growth rate, entrainment 
and mixing can be used from the theory of turbulent jets. When the ambient fluid is flowing at right angles to the discharge, the plume will quickly be bent over in the direction of the cross flow. However, the initial buoyancy and momentum will continue to be important in the dynamics of the plume.

In the far field, diffusion is accomplished by the ambient turbulence, although the initial momentum and buoyancy may still be important. When the ambient fluid is stably stratified, the diluted effluent will reach a level where its density equals that of the ambient fluid, and will spread almost entirely in the horizontal direction. The spread of a passive material in a flowing or stratified, turbulent medium has been reviewed extensively [69-71, 5].

The work on plumes is divided into two groups by application, atmospheric plumes and discharges in water. Each application has particular, interesting problems and an extensive literature associated with it. For example, in the atmosphere the initial buoyancy is determined not only by the temperature, but also by the average molecular weight and the particulate content of the discharged gas. Whereas the gas will generally rise and mix as described above, the particles will settle out at a rate dependent upon their mass and size distribution. When water vapor is present, condensation, with an associated heat release and increase in buoyancy, can take place. In addition, chemical reactions, and particularly photochemistry (induced by the sun), are important in consideration of atmospheric pollutants. Therefore, the equations describing the chemical kinetics must be carried along with the equations for the plume dynamics. Finally, in engineering design, stack height and placement are important for consideration of the dispersion of effluent material.

Similar problems must be considered when thermal discharges in water are analyzed. However, some of the problems and the emphasis of the problems are different. In a water body, a surface is present and this can alter the dispersion dynamics greatly. In addition, as described earlier, a distinctive thermal structure including a thermocline, is often present, trapping the discharge at a particular level and causing two-dimensional, horizontal spreading. If the discharge does reach the surface, the spreading will be two-dimensional and heat will be lost to the atmosphere directly and through evaporation. From the engineering-design viewpoint, the position of the water intake and discharge and the design of the diffuser at the discharge are important.

\section{Some Important Problem Areas}

In this section some important problems related to stratified and rotating fluids will be discussed. The problems can be broadly classed into four categories, turbulence, nonlinear processes, numerical computation of flows and applications. Each category will be discussed briefly; as in preceding sections, no attempt will be made to be complete. The emphasis will be on theory, but it should be noted that proper understanding arises only when theory and experiment supplement each other.

Turbulence remains one of the most difficult and challenging phenomena in fluid mechanics. Because it is a nonlinear, random process, the progress in understanding and analyzing it has been slow. Some years back there was a great deal of research activity and several books summarizing the status. Some of this work concerned homogeneous, isotiopic turbulence and some turbulent shear flows.

Recently, there has been an increase in interest again, and the emphasis has now been on turbulent shear flows and on turbulent mixing in the presence of stratification. Both of these aspects of turbulence enjoy wide applicability, as seen in this review. They also have another feature in common: the mean fluid properties are anisotropic. In shear flow, a direction is determined by the vorticity of the mean flow. In stratified fluids, a direction is determined by gravity. The anisotropy might be expected to affect the turbulent eddies in fundamental ways: eddies with their vorticity parallel to the direction determined by the mean properties will be influenced differently than those perpendicular to the mean properties. With the resurgence of interest in turbulence, it is anticipated that many of the problems associated with turbulence in an anisotropic medium will be addressed, and progress in understanding it will be made.

Early in this report it was noted that linear problems have received much more attention than nonlinear ones because the tools of linear analysis are more highly developed. The analysis of nonlinear problems has always required elements of ingenuity, patience and luck, and individual problems generally require unique approaches. However, an increased number of solutions to nonlinear problems has increased the variety of available approaches to these problems. Some approaches can be used directly or generalized to attack other problems. So, many areas of nonlinear analysis are developing. In particular, nonlinear waves, convection and motions in rotating or stratified fluids are all being examined to an increasing extent. 
Continued effort in these areas is needed. In addition, the methods of analysis should be classified and generalized to aid the attack on other nonlinear problems.

With the advent of high-speed computers, numerical solutions to a wide variety of physical problems have become a reality. As a result, computational physics and computational fluid mechanics have become established branches of theoretical physics and theoretical mechanics. Finite difference techniques have been developed to integrate ordinary differential equations and initial-value and boundary-value problems associated with partial differential equations. Although enormous effort has been expended in recent years in developing and increasing the efficiency of numerical techniques, more is needed. With the guidance of car eful analysis, the computation of fluid flows, especially stratified and rotating flows, should be pursued vigorously.

Finally, the application to physical problems should be made of the basic results devermined for stratified and rotating fluids. Much work has already been performed along this line, and some of it was discussed in the preceding section. However, many problems still remain in the earth sciences, in astrophysics and in more technologically oriented areas such as those discussed in section 7.4 .

\section{Summary}

A survey has been presented of phenomena in stratified and rotating fluids. Care was taken to define important quantities, to discuss basic concepts, to derive the fundamental equations and to present the basic nondimensional parameters associated with these flows. After a rather extensive section on waves, the analogy between stratified and rotating flows was discussed. Then nonlinear processes, and transport and diffusion processes were reviewed. Although this report is rather brief in parts, it displays the rich variety of phenomena in stratified and rotating fluids. It also tabulates many of the important references.

This report also contains a discussion of some applications. Physical oceanography, physical limnology and meteorology are all areas of application in the earth sciences. The variety of applications was stressed at the expense of depth and completeness. The more technological application of plumes in bodies of water or in the atmosphere was also discussed.

Finally, some important problem areas were briefly surveyed. These areas are turbulence, nonlinear processes, numerical computation of flows and further applications.

I wish to express my gratitude to Burton C. Colvin and to Hans J. Oser of the Applied Mathematics Division of NBS for providing me the opportunity to write this report. Appreciation is also due Professor C. C. Lin of the Massachusetts Institute of Technology.

I want to thank Mrs. Regina Kopec for carefully typing and correcting this long manuscript and Howard Baum of the Fire Science Division and Ronald Davis of the Engineering Mechanics Division of NBS for reading it and giving helpful comments.

\section{References}

[1] Tennekes, H., The atmospheric boundary layer, Physics Today, pp. 52-63, Jan. 1974.

[2] Revelle, R., The ocean, Scientific American (special issue on the ocean) 221, pp. 54-65 (Sept. 1969).

[3] Spilhaus, A. F., Jr., Geophysics-An overview, Physics Today 27, pp. 23-26 (March 1974); also G. Schubert and O. L. Anderson; The earth's thermal gradient, Physics Today 27, pp. 28-34 (March 1974).

[4] Turner, J. S., Buoyancy Effects in Fluids (Cambridge University Press, Cambridge, England, 1973).

[5] Phillips, O. M., The Dynamics of the Upper Ocean (Cambridge University Press, Cambridge, England, 1966).

[6] Eckart, C., Hydrodynamics of Oceans and Atmospheres (Pergamon Press, New York, 1960).

[7] Yih, C.-S., Stratified Flows, Ann. Rev. of Fluid Mechan. 1, 73-110 (1969); also C. S., Yih, Dynamics of Nonhomogeneous Fluids (Macmillan, New York, 1965).

[8] Harleman, D. F., Stratified Flow, Chap. 26, Handbook of Fluid Dynamics, Edited by V. L. Streeter (McGraw-Hill, New York, 1961).

[9] Greenspan, H. P., The Theory of Rotating Fluids (Cambridge University Press, Cambridge, England, 1968).

[10] Lighthill, M. J., Dynamics of rotating fluids: A survey, J. Fluid Mech. 26, 411-431 (1966).

[11] Panofsky, H. A., The atmospheric boundary layer below 150 meters, Ann. Rev. Fluid Mech. 6, 147-177 (1974).

[12] Maxworthy, T., and Browand, F. K., Experiments in rotating and stratified flows: Oceanographic application, Ann. Rev. Fluid Mech. 7, 273-305 (1975).

[13] Csanady, G. T., Hydrodynamics of large lakes, Ann. Rev. Fluid Mech. 7, 357-386 (1975).

[14] Benton, E. R., and Clark, A., Jr., Spin-up, Ann. Rev. Fluid Mech. 6, 257-386 (1974). 
[15] Schwarzschild, M., Structure and Evolution of the Stars (Princeton University Press, Princeton, New Jersey, 1958).

[16] Veronis, G., The analogy between rotating and stratified fluids, Ann. Rev. Fluid Mech. 2, 37-66 (1970).

[17] Lighthill, M. J., Studies on magneto-hydrodynamic waves and other anisotropic wave motions, Phil. Trans. Roy. Soc. London, Series A, 252, Math. \& Phys. Sciences, pp. 397-430, (1959).

[18] Lighthill, M. J., Waves in fluids, Comm. Pure \& Appl. Math. 20, 267-293 (1967).

[19] Hutchinson, G. E., A Treatise on Limnology, Vol. I (Wiley, New York, 1960).

[20] Liepmann, H. W., and Roshko, A., Elements of Gasdynamics (Wiley, New York, 1960).

[21] Batchelor, G. K., The conditions for dynamical similarity of motions of a frictionless perfect-gas atmosphere, Quarterly J. Roy. Meteorological Soc. 79, 224-235 (1953).

[22] Sundaram, T. R., Stratton, J. E., and Rehm, R. G., Turbulent wakes in a stratified medium, Calspan Report CAL-A G-3018-A-1, (Nov. 1971).

[23] Sundaram, T. R., Rehm, R. G., Rudinger, G., and Merritt, G. E., Research on the Physical Aspects of Thermal Pollution, Water Pollution Control Series 16/30 DPU 02/71, Environmental Protection Agency Water Quality Office (Feb. 1971); also see reference [57].

[24] Lighthill, M. J., Group velocity, J. Inst. Math. Its Appl. 1, 1-28 (1965).

[25] Lighthill, M. J., On waves generated in dispersive systems by travelling forcing effects, with applications to the dynamics of rotating fluids, J. Fluid Mech. 27, 725-752 (1967).

[26] Whitham, G. B., Linear and Nonlinear Waves (Wiley, New York, 1974).

[27] Görtler, H., Über eine Schwingungserscheinung in Flüssigkeiten mit Stabiler Dichteschichtung, Z. Angew. Math. Mech. 23, 65-71 (1943).

[28] Mowbray, D. E., and Rarity, B. S. H., A theoretical and experimental investigation of the phase configuration of internal waves of small amplitude in a density stratified liquid, J. Fluid Mech. 28, 1-16 (1967).

[29] Miles, J. W., Internal waves generated by a horizontally moving source, Geophysical Fluid Dynamics 2 , 63-67 (1971).

[30] Carrier, G. F., and Chen, A., Internal Waves Produced by Underwater Vehicles, TR W Report 182026001-RO-00, Vol. I (1971).

[31] Stevenson, T. N., Some two-Dimensional Internal Waves in a Stratified Fluid, J. Fluid Mech., pp. 715720 (1968).

[32] Schooley, A. H., and Stewart, R. W., Experiments with a Self-Propelled Body Submerged in a Fluid with a Vertical Density Gradient, J. Fluid Mech. 15, 83 (1963); also A. H. Schooley, Wake collapse in a stratified fluid, Science 157, 421 (1967).

[33] Schooley, A. H., Wake collapse in a stratified fluid: Experimental exploration of scaling characteristics, Science 160, 763 (1968).

[34] Merritt, G. E., Wake growth and collapse in a stratified flow, AIAA Journal 12, 940-949 (1974).

[35] Wu, J., Mixed region collapse with internal wave generation in a density-stratified medium, J. Fluid Mech. 35, 531, (1969).

[36] Hendershott, M. C., Impulsively started oscillations in a rotating, stratified fluid, J. Fluid Mech. 36, 513-527 (1969).

[37] Hurley, D. G., The Emission of internal waves by vibrating cylinders, J. Fluid Mech. 36, 657-672 (1969).

[38] McLaren, T. I., Pierce, A. D., Fohl, T., and Murphy, B. L., An investigation of internal gravity waves generated by a buoyantly rising fluid in a stratified medium, J. Fluid Mech. 57, 229-240 (1973).

[39] Rehm, R. G., and Radt, H. S., Jr., Internal Waves Generated by a Translating, Oscillating Body, J. Fluid Mech. 68, 235 (1975).

[40] Stevenson, T. N., and Thomas, N. H., Two-dimensional internal waves generated by a travelling oscillating cylinder, J. Fluid Mech. 36, 505-511 (1969); also, T. N. Stevenson, Axisymmetric internal waves generated by a travelling oscillating body, J. Fluid Mech. 35, 219-224 (1969).

[41] Görtler, H., Einige Bemerkungen über Strömungen in rotierenden Flüssigkeiten, Z. Angew. Math. Mech. 24, 210-214 (1944).

[42] Oser, H., Erzwungene Schwingungen in rotierenden Flüssigkeiten, Archive Rational Mech. \& Analysis 1, 81-96 (1957).

[43] Oser, H., Experimentelle Untersuchung über harmonische Schwingungen in rotierenden Flüssigkeiten, Z. Angew. Math. Mech. 38, 386-391 (1958).

[44] Prabhakara Rao, G. V., Waves generated in rotating fluids by travelling forcing effects, J. Fluid Mech. 61, 129-158 (1973); also V. Subba Rao and G. V. Prabhakara Rao; On waves generated in rotating stratified liquids by travelling forcing effects 46, 447-464 (1971).

[45] Long, R. R., Finite amplitude disturbances in the flow of inviscid, rotating and stratified fluids over obstacle, Ann. Rev. Fluid Mech. 4, 69-92 (1972).

[46] Long, R. R., Some aspects of the flow of stratified fluids, I. A theoretical investigation, Tellus V $42-57$ (1953).

[47] Stoker, J. J., Water Waves (Interscience Publishers, 1957), chapter 10.

[48] Lick, W., Nonlinear wave propagation in fluids, Ann. Rev. Fluid Mech. 2, 113-136 (1970). 
[49] Phillips, O. M., Nonlinear dispersive waves, Ann. Rev. Fluid Mech. 6, 93-110 (1974).

[50] Brueckner, K. A., and Jorna, S., Laser-driven fusion, Rev. Mod. Phys. 46, 325-367 (1974).

[51] Barenblatt, G. I., and Zeldovich, Ja. B., Self similar solutions as intermediate asymptotic, Ann. Rev. Fluid Mech. 4, 285-312 (1972); also L. I. Sedov, Similarity and Dimensional Methods in Mechanics (Academic Press, New York, 1959).

[52] Chandrasekhar, S., Hydrodynamic and Hydromagnetic Stability, (Oxford Press, London, 1961).

[53] Priestly, C. H. B., Turbulent Transfer in the Lower Atmosphere, (Chicago U. Press, 1959).

[54] Sutton, O. G., Micrometeorology (McGraw-Hill, New York, 1953).

[55] Lumley, J. L., and Panofsky, H. A., The Structure of Atmospheric Turbulence (Interscience, New York, 1964).

[56] Monin, A. S., The atmospheric boundary layer, Ann. Rev. Fluid Mech. 2, 225-250 (1970).

[57] Sundaram, T. R., and Rehm, R. G., The seasonal thermal structure of deep temperate lakes, Tellus XXV, 157-168 (1973).

[58] Robinson, A. R., Oceanography, Research Frontiers in Fluid Mechanics, R. J. Seeger and G. Temple editors (Interscience, New York, 1965). pp. 504-533.

[59] Engel, L., The Sea, Life Nature Library (Time Incorporated, New York, 1963).

[60] Stewart, R. W., The atmosphere and the ocean, Scientific American 221, 76-87 (Sept. 1969).

[61] Robinson, A. R., Boundary layers in ocean circulation models, Ann. Rev. Fluid Mech. 2, 293-312 (1970).

[62] Hendershott, M., and Munk, W., Tides, Ann. Rev. Fluid Mech. 2, 205-224 (1970).

[63] Sutton, O. G., Meteorology, Research Frontiers in Fluid Mechanics, Eds. R. J. Seeger and G. Temple (Interscience, New York, 1965), pp. 482-503.

[64] Phillips, N. A., Models for weather prediction, Ann. Rev. Fluid Mech. 2, 251-292 (1970).

[65] Harlemen, D. R. F., and Stolzenbach, K. D., Fluid mechanics of heat disposal from power generation, Ann. Rev. Fluid Mech. 4, 7-32 (1972).

[66] Fay, J. A., Buoyant plumes and wakes, Ann. Rev. Fluid Mech. 5, 151-159 (1973).

[67] Koh, R. C. Y., and Brooks, N. H., Fluid mechanics of waste-water disposal in the ocean, Ann. Rev. Fluid Mech. 7, 187-212 (1975).

[68] Fay, J. A., and Hoult, D. P. (editors), Aerophysics of Air Pollution, (AIAA Selected Reprints IX, AIAA, New York, 1969).

[69] Pasquill, F., Atmospheric Diffusion (Van Nostrand, Princeton, New Jersey, 1962).

[70] Okubo, A., A Review of Theoretical Models for Turbulent Diffusion in the Sea, J. Oceanographical Society of Japan, 20th Anniversary Volume, (1962), pp. 286-320.

[71] Fischer, H. B., Longitudinal dispersion and turbulent mixing in open channel flow, Ann. Rev. Fluid Mech. 5, 59-78 (1973).

[72] Howard, L. N., Fundamentals of the theory of rotating fluids, J. Appl. Mech. 30, 481-485 (1963).

[73] Taylor, G. I., Experiments on the motion of solid bodies in rotating fluids, Proc. Roy. Soc. Series A, 104, 213-218 (1923).

(Paper 80B3-451) 